OPEN ACCESS

Edited by:

Sapna Patel,

University of Texas MD Anderson

Cancer Center, United States

Reviewed by:

Scott X Atwood,

University of California, Irvine,

United States

Rogelio González Sarmiento, University of Salamanca, Spain

${ }^{*}$ Correspondence:

Piotr Kozlowski

kozlowp@ibch.poznan.pl

Specialty section:

This article was submitted to

Skin Cancer,

a section of the journal

Frontiers in Oncology

Received: 03 August 2021 Accepted: 08 November 2021 Published: 25 November 2021

Citation:

Nawrocka PM, Galka-Marciniak P,

Urbanek-Trzeciak MO,

M-Thirusenthilarasan I, Szostak N,

Philips A, Susok L, Sand $M$ and Kozlowski P (2021) Profile of Basal Cell

Carcinoma Mutations and Copy

Number Alterations - Focus on GeneAssociated Noncoding Variants.

Front. Oncol. 11:752579. doi: 10.3389/fonc.2021.752579

\section{Profile of Basal Cell Carcinoma Mutations and Copy Number Alterations - Focus on Gene- Associated Noncoding Variants}

\author{
Paulina Maria Nawrocka ${ }^{1}$, Paulina Galka-Marciniak ${ }^{1}$, Martyna Olga Urbanek-Trzeciak ${ }^{1}$, \\ Ilamathi M-Thirusenthilarasan ${ }^{1}$, Natalia Szostak ${ }^{2}$, Anna Philips ${ }^{2}$, Laura Susok ${ }^{3}$, \\ Michael Sand ${ }^{3,4}$ and Piotr Kozlowski ${ }^{1 *}$ \\ 1 Department of Molecular Genetics, Institute of Bioorganic Chemistry, Polish Academy of Sciences, Poznan, Poland, \\ 2 Laboratory of Bioinformatics, Institute of Bioorganic Chemistry, Polish Academy of Sciences, Poznan, Poland, ${ }^{3}$ Department \\ of Dermatology, Venereology and Allergology, St. Josef Hospital, Ruhr-University Bochum, Bochum, Germany, ${ }^{4}$ Department \\ of Plastic Surgery, St. Josef Hospital, Catholic Clinics of the Ruhr Peninsula, Essen, Germany Department of Plastic, \\ Reconstructive and Aesthetic Surgery, St. Josef Hospital, Essen, Germany
}

Basal cell carcinoma (BCC) of the skin is the most common cancer in humans, characterized by the highest mutation rate among cancers, and is mostly driven by mutations in genes involved in the hedgehog pathway. To date, almost all BCC genetic studies have focused exclusively on protein-coding sequences; therefore, the impact of noncoding variants on the BCC genome is unrecognized. In this study, with the use of whole-exome sequencing of 27 tumor/normal pairs of BCC samples, we performed an analysis of somatic mutations in both protein-coding sequences and gene-associated noncoding regions, including 5'UTRs, 3'UTRs, and exon-adjacent intron sequences. Separately, in each region, we performed hotspot identification, mutation enrichment analysis, and cancer driver identification with OncodriveFML. Additionally, we performed a whole-genome copy number alteration analysis with GISTIC2. Of the $>80,000$ identified mutations, 50\% were localized in noncoding regions. The results of the analysis generally corroborated the previous findings regarding genes mutated in coding sequences, including PTCH1, TP53, and MYCN, but more importantly showed that mutations were also clustered in specific noncoding regions, including hotspots. Some of the genes specifically mutated in noncoding regions were identified as highly potent cancer drivers, of which BAD had a mutation hotspot in the 3'UTR, DHODH had a mutation hotspot in the Kozak sequence in the 5'UTR, and CHCHD2 frequently showed mutations in the 5'UTR. All of these genes are functionally implicated in cancer-related processes (e.g., apoptosis, mitochondrial metabolism, and de novo pyrimidine synthesis) or the pathogenesis of UV radiation-induced cancers. We also found that the identified $B A D$ and $C H C H D 2$ mutations frequently occur in melanoma but not in other cancers via The Cancer Genome Atlas analysis. Finally, we identified a frequent deletion of chr9q, encompassing $P T C H 1$, and 
unreported frequent copy number gain of chr9p, encompassing the genes encoding the immune checkpoint ligands PD-L1 and PD-L2. In conclusion, this study is the first systematic analysis of coding and noncoding mutations in BCC and provides a strong basis for further analyses of the variants in BCC and cancer in general.

Keywords: basal cell carcinoma (BCC), cancer somatic mutations, noncoding mutations, immune checkpoint, copy number alterations, cancer drivers, TERT, DPH3

\section{INTRODUCTION}

Basal cell carcinoma (BCC), a type of nonmelanoma skin cancer, is the most common human cancer affecting predominantly elderly people of the Caucasian population (1-3). The lifetime risk of BCC in the Caucasian population is $\sim 30 \%$, and it is higher in men and fair-skinned people. BCC usually occurs sporadically but can also develop as a result of Gorlin syndrome (also known as nevoid basal cell carcinoma syndrome), an autosomal dominant hereditary condition with an incidence of approximately $1: 30,000$ (4) characterized by the frequent appearance of multiple BCC lesions that develop at a younger age together with skeletal abnormalities, odontogenic keratocysts, and an increased risk of medulloblastoma. Histologically, BCCs are classified into three major subtypes: nodular, which is the most common subtype; superficial; and infiltrative or sclerodermiform. Other subtypes as well as mixed types occur less frequently (5-7). Predominantly, superficial and nodular BCCs are slow-growing, locally invasive, epidermal tumors with a metastasis rate of $<0.1 \%$ $(8,9)$, while infiltrative BCCs are characterized by more aggressive, tong-like, subclinical growth patterns mimicking icebergs, as they often grow below clinically healthy-looking skin $(10,11)$. Although BCC aggressiveness and metastatic potential are overall low, the commonness of BCC and the increasing incidence associated predominantly with aging populations has brought attention to its pathogenesis $(2,3,12-17)$. Exposure to ultraviolet (UV) radiation, which can lead to point mutations frequently represented by $\mathrm{C}>\mathrm{T}$ and $\mathrm{CC}>\mathrm{TT}$ transitions, is the main causative factor in the pathogenesis of BCC (18). Additional risk factors include ionizing radiation, arsenic ingestion, and immune suppression $(19,20)$.

BCC is characterized by the highest mutation rate observed among cancers, having over 65 mutations/Mbp $(14,15)$. The most frequent genetic alterations occurring in BCC are mutations disturbing the hedgehog ( $\mathrm{SHH} / \mathrm{PTCH} 1 / \mathrm{SMO}$ ) pathway, predominantly loss-of-function mutations in PTCH1 but also activating mutations in SMO; these genes encode two transmembrane proteins, PTCH1 (also known as Patched1) and SMO (also known as Smoothened), respectively $(14,15)$. The pathway is activated by the $\mathrm{SHH}$ signaling protein (also known as Sonic hedgehog), which binds to the extracellular domain of PTCH1, disabling inhibition of SMO; this in turn activates GLI transcription factors. Germline mutations in $\mathrm{PTCH} 1$ predispose patients to Gorlin syndrome (21).

Previous studies, including whole-exome sequencing (WES) analyses, have also recognized other genes/pathways frequently mutated in BCC, including TP53, MYCN, PPP6C, PTPN14, STK19, and LATS1 $(14,15)$, as well as genes involved in the RTK-RAS-PI3K and Hippo-YAP pathways (15). However, as an overwhelming majority of BCC genetic studies (as well as those in other cancers) have focused almost exclusively on protein-coding sequences, very little is known about mutations in noncoding regions (noncoding mutations). Noncoding mutations are not studied/reported even if detected, e.g., as a result of WES. On the other hand, it is well known that the noncoding parts of genes, i.e., promoters, introns, or 5' and 3' untranslated regions (5'UTRs and 3'UTRs, respectively), encompass numerous functional elements important for the proper functioning of the genes (22-24). Somatic mutations may disrupt or modify the properties of these elements, acting either as gain- or loss-of-function mutations and thus enhancing/accelerating or switching off the function of some genes. Despite the limited number of studies on noncoding mutations, there are some spectacular examples of noncoding driver mutations, for example, TERT promoter mutations, which occur most frequently in melanoma, brain, and bladder cancers but are also reported in BCC (25-27), and mutations in the precursor of miR-142, which frequently occur in non-Hodgkin lymphomas and acute myeloid leukemia [summarized in (28)]. The miRNA biogenesis enzyme DICER has also been shown to bear mutations that could play a role in aberrant miRNA expression in BCC (29-31). It should also be noted that an effort to catalog cancer somatic mutations in the noncoding genome has recently been undertaken $(32,33)$; however, this pancancer project does not include BCC.

To preliminarily explore the occurrence of noncoding somatic mutations in BCC, we performed WES of over two dozen BCC samples, extending the analysis beyond protein-coding sequences and focusing on gene-associated noncoding regions, i.e., 5'UTRs, 3'UTRs, and exon-adjusted sequences of introns, covered by standard WES approaches. Apart from the fact that our results well-replicate those of previous BCC studies in terms of mutations in protein-coding genes, we showed that a substantial portion of mutations is located in noncoding regions. Many of these mutations frequently recur in particular noncoding regions or in specific hotspot positions. Computational analyses showed that some of the gene mutations in noncoding regions are potential cancer drivers and are functionally related to skin cancers. Additionally, whole-genome copy number alteration (CNA) analysis revealed frequent deletion of $\operatorname{chr} 9 \mathrm{q}$, encompassing PTCH1, and unreported frequent amplification of chr9p, including the genes encoding two immune checkpoint ligands PD-L1 and PD-L2. 


\section{MATERIALS AND METHODS}

\section{Sample Collection and DNA Preparation}

A total of 27 pairs of tissue (tumor and normal adjacent healthy skin) were collected from the Department of Plastic Surgery, St. Josef Hospital, Catholic Clinics of the Ruhr Peninsula, Essen, Germany. While excising the BCC tissues with cold steel under local anesthesia, 4-mm punch biopsies were taken from the center of the tumor and from nonlesional epithelial skin (as normal, intraindividual controls). These samples were immediately placed in RNAlater (Qiagen, Hilden, Germany) and stored at $-80^{\circ} \mathrm{C}$. Tissue homogenization was performed with stainless steel beads of $5 \mathrm{~mm}$ (Qiagen) and TissueLyser LT (Qiagen). DNA was extracted with an AllPrep DNA/RNA/ miRNA Universal Kit (Qiagen) according to the manufacturer's protocol. All samples were quantified using a NanoDrop One (Thermo Scientific, Waltham, USA) and Qubit fluorometer 3.0 (Invitrogen) (Qubit dsDNA HS Assay (Life Technologies, Carlsbad, USA)), and DNA size and quality were tested using gel electrophoresis.

\section{Exome Sequencing and Data Processing}

The library was prepared with $200 \mathrm{ng}$ of high-quality DNA using the SureSelectXT Library Prep Kit (Agilent). A SureSelectXT Human All Exon V6 kit (Agilent) was used for exome capture. Sequencing was performed on an Illumina NovaSeq 6000 (San Diego, USA), generating $2 \times 100$ bp paired-end reads. Library preparation, exome enrichment, and sequencing were performed at CeGaT, Tuebingen, Germany. Demultiplexing of the sequencing reads was performed with Illumina bcl2fastq (2.19). Adapters were trimmed with Skewer (version 0.2.2) (34). The Phred score was given with Illumina standard Phred encoding (offset +33). For each sample, two FASTQ files corresponding to forward and reverse reads were obtained. Next steps were done by us on the Poznan Supercomputing and Networking Center (PSNC) Eagle supercomputer. Paired-end reads were aligned to hg38 using BWA. PCR duplicates were marked and removed with the Picard package. Indel realignments with known sites and base quality score recalibration were performed with GATK version 4.1.2.0. SAM to BAM conversion was done using SAMtools. Somatic single-nucleotide variants were called with MuTect2 (version 4.1.0.0. with the use of the tumor-normal mode). Additionally, to avoid false-positive somatic mutations, we performed filtering for germline variants present in the gnom $A D$ database (version 2.1.1). We also generated and flagged variants with a panel of normals (PoN) comprising variants representing commonly occurring sequencing noise that may mimic low allele-fraction somatic variants. We also added information about the localization of mutations in gene subregions (CDS, 5'UTR, 3'UTR, or introns) by use of an inhouse Python script. From the list of somatic mutations, we additionally removed those that did not fulfill the following criteria: (i) at least five alternative allele-supporting reads in a tumor sample; (ii) frequency of alternative allele-supporting reads in a tumor sample of at least 0.05 ; and (iii) frequency of alternative allele-supporting reads in the tumor sample at least $5 \times$ higher than that in the corresponding normal sample.

\section{Validation of Mutations and Sequencing of the TERT and DPH3 Promoters}

A panel of 51 mutations detected by WES was validated by Sanger sequencing of the appropriate PCR fragments amplified with primers shown in Table S1. The primers used for amplification and sequencing of the TERT and DPH3 promoters are shown in Table S1. All fragments were sequenced in two directions with the BigDye v3.1 kit (Applied Biosystems, Foster City, CA, USA), and the sequencing reactions were separated with capillary electrophoresis (POP7 polymer; ABI Prism 3130xl apparatus; Applied Biosystems, Foster City, CA, USA) according to the standard manufacturer's recommendations.

\section{Mutational Signature Analysis}

To analyze mutational signatures, we used the web application Mutational Signatures in Cancer [MuSiCa; http://bioinfo. ciberehd.org/GPtoCRC/en/tools.html (35)], allowing the visualization of the somatic mutational profile of each analyzed sample and estimation of the contribution values of the predefined mutational signatures [(36); Catalogue Of Somatic Mutations In Cancer, COSMIC 2020]. Samples BCC14 and BCC21 were excluded from the signature analysis due to an insufficient number of mutations.

\section{Identification of Hotspots, Frequently Mutated Genes, and Cancer Drivers}

We defined genomic positions mutated in at least $3(>10 \%)$ samples as hotspots. Mutations occurring in directly adjacent nucleotides were merged into one hotspot.

We defined genes with nonsynonymous mutations in a coding region in at least 5 samples, with mutations in a $5^{\prime} \mathrm{UTR}$, in at least 4 samples, with mutations in a $3^{\prime}$ UTR in at least 4 samples, and with mutations in introns (up to $40 \mathrm{nt}$ from exon/ intron boundaries) in at least 5 samples as frequently mutated. From the analysis, we excluded genes known to be commonly hypermutated with passenger mutations as a result of the increased background mutation rate but not related to cancer, listed in (37). To distinguish synonymous from nonsynonymous mutations, we used the SnpEff - genetic variant annotation and functional effect prediction toolbox (38), available on the Subio platform (Subio, Inc., Kagoshima, Japan, http://www.subio.jp). We also considered splice-site mutations located in introns up to $+/-2$ nt from exons as coding region mutations.

OncodriveFML (39) was run using the CADD score (hg38, version 1.6). The signature method was set as a complement, the statistical method was set to "amean", and indels were included in the analysis using a max method (max_consecutive was set to 7 as default).

\section{Copy Number Analysis}

To identify chromosome arm-level and focal regions that were significantly amplified or deleted, we used GISTIC2 (40) with the following parameters: threshold for copy number amplifications and deletions, 0.2 ; confidence level to calculate the region containing a driver, 0.9 ; broad-level analysis; and the arm peel method to reduce noise. 
To validate CNAs involving chromosome 9, i.e., chr9p duplications/amplifications (affecting JAK2, PDL1/CD274, and PDL2/CD273) and chr9q deletions (affecting PTCH1), we designed and generated an MLPA assay covering the entire chromosome 9. In total, the assay consisted of 20 probes, including (i) 7 probes distributed over the chr9p $(n=5)$ and chr9q $(\mathrm{n}=2)$ arms, 2 probes located in or in close proximity to JAK2, PDL2, $P D L 1$, and PTCH1 (in total 8 gene-specific probes), and 5 control probes (located on different chromosomes outside of chromosome 9 and regions of known cancer-related genes). The sequences and detailed characteristics of all probes as well as their exact positions are shown in Table S2.

The MLPA probes and the probe-set layout were designed according to a previously proposed and well-validated strategy $(41,42)$. Shortly, each probe was composed of two half-probes of equal size, and the total probe length ranged from 93 to $172 \mathrm{nt}$. The target sequences for the probes were selected to avoid common SNPs, repeat elements, and sequences of extremely high or low GC content. The MLPA probes were synthesized by IDT (Skokie, IL, USA). The MLPA reactions were run according to the manufacturer's general recommendations (MRC-Holland, Amsterdam, the Netherlands). All reagents except the probe mixes were purchased from MRC-Holland (http://www.mlpa. com). The products of the MLPA reaction were subsequently diluted 20x in HiDi formamide containing GS Liz600, which was used as a DNA sizing standard, and separated via capillary electrophoresis (POP7 polymer) in an ABI Prism 3130XL apparatus (Applied Biosystems, Carlsbad, CA, USA). The obtained electropherograms were analyzed using GeneMarker software v2.4.0 (SoftGenetics, State College, PA, USA). For each individual sample, the signal intensity of each probe was divided by the geometric average signal intensity of the control probes to normalize the run-to-run signal variation, and then the normalized signal of each probe in cancer samples was divided by the corresponding signal in the corresponding normal samples and multiplied by 2 . The final MLPA result of each sample is presented on a bar-plot, in which the bars show the relative copy number value of the subsequent probes.

\section{TCGA Analysis}

To compare the mutations recurring in BCC with mutations in other cancers, we used WES-generated somatic mutation datasets of 10,369 samples representing 33 cancer types generated and deposited in the TCGA repository (http:// cancergenome.nih.gov). The full names and abbreviations of all TCGA cancer types are shown in Table S3. Somatic mutations were identified against matched normal samples with the use of the standard TCGA pipeline (including the Mutect2, Muse, Varscan, and SomaticSnipper algorithms). We extracted somatic mutation calls (with PASS annotation only) localized in the annotated exons of BAD, DHODH, CHCHD2, FLG, and FLG2 (exon sequences were extended by $2 \mathrm{nt}$ to enable identification of intronic splice-site mutations). The extraction was performed as described in our earlier study (43) with a set of in-house Python scripts available at (https:/github.com/ martynaut/mirnaome_somatic_mutations).

\section{Mutations Visualization}

All mutations were annotated according to HGVS nomenclature (at the transcript and protein levels), and the effects of mutations were defined using the Ensembl Variant Effect Predictor (VEP) tool. For visualization of mutations on gene maps, we used ProteinPaint from St. Jude Children's Research Hospital - PeCan Data Portal (44). The protein domains visualized on gene maps were positioned according to UniProt data (45). The comutation plot showing frequently mutated genes was created with the use of the Python library CoMut (46).

\section{Analysis of RNA Regulatory Motifs}

Target predictions were performed with the TargetScan Custom (release 5.2) web tool (47). The secondary RNA structures were predicted using mfold software (48) with default parameters. RNA sequence/structure functional motifs and transcription factor binding sites were analyzed with the RegRNA 2.0 (49) and MotifMap (50) web tools.

\section{Statistics}

Specific statistical tests are indicated in the text, and a $p$-value $<0.05$ was considered significant. If necessary, $p$-values were corrected for multiple tests with the Benjamini-Hochberg procedure.

\section{RESULTS}

\section{Overall Sequencing and Mutation Occurrence Characterization}

We performed WES on 27 paired tumor and corresponding intraindividual control skin DNA samples isolated from 22 nodular and 5 superficial BCC subtypes and corresponding healthy skin tissue. The average coverage of the targeted regions was $183 \mathrm{x}$ (185x in normal and 180x in tumor samples), ranging in different samples from $134 x$ to $232 x$. In total, we identified 84,571 cancer-sample-specific somatic mutations (Table S4), of which 42,380 (50.1\%) were located in protein-coding (coding) regions, and the remaining 42,191 (49.9\%) were located in noncoding regions (Table 1 and Figure 1A). The noncoding regions included (i) 5'UTRs, (ii) 100 bp fragments of 3'UTRs adjacent to coding sequences (3'UTRs), (iii) exon-adjacent $\sim 100$ bp fragments of introns (introns), and (iv) sequences other than those classified above (i-iii), mostly intergenic sequences located upstream and downstream of the first and last gene exons (intergenic regions) (51). The average coverage of the mutated positions was $169 \mathrm{x}$ and was slightly higher in coding $(195 \mathrm{x})$ than in noncoding regions $(142 \mathrm{x})$, whereas the average fraction of reads mapping to alternative alleles was 0.35 (0.33 in coding and 0.40 in noncoding regions). The average mutation rate calculated based on the coding regions was 52.8 mutations/Mbp (ranging from 0.1 to 287.5), which, although slightly lower than that observed before in BCC $(15,52)$, is still higher than that in any other tested cancer type. Although somewhat counterintuitive, the lower mutation burden in our study than in other BCC studies $(15,52)$ may result from the much higher sequencing coverage in our study, which gave us much higher statistical power to filter out the fraction of 
TABLE 1 | Summary of somatic mutation distribution and mutation types in BCC.

\begin{tabular}{|c|c|c|c|c|c|c|}
\hline Genomic regions & $\begin{array}{l}\text { No. }(\%) \text { of } \\
\text { mutations }\end{array}$ & $\begin{array}{l}\text { Average coverage of } \\
\text { mutation positions }\end{array}$ & Average alternative allele fraction & $\begin{array}{c}\text { No. }(\%) \text { of } \\
\text { substitutions }\end{array}$ & $\begin{array}{c}\text { No. }(\%) \text { of double } \\
\text { substitutions }\end{array}$ & No. $(\%)$ of short indels \\
\hline all mutations & $84,571(100)$ & 169 & 0.35 & $79,960(94.5)$ & $3128(3.7)$ & $1483(1.8)$ \\
\hline coding & $42,380(50.1)$ & 195 & 0.33 & $40,503(95.6)$ & $1697(4.0)$ & $180(0.4)$ \\
\hline noncoding & 42,191 (49.9) & 142 & 0.37 & 39,457 (93.5) & $1431(3.4)$ & $1303(3.1)$ \\
\hline introns & 32,805 (38.8) & 136 & 0.38 & $30,655(93.4)$ & $1076(3.3)$ & $1074(3.3)$ \\
\hline 3'UTR & $2,926(3.5)$ & 154 & 0.39 & $2,721(93.0)$ & $100(3.4)$ & $105(3.6)$ \\
\hline 5'UTR & $2,832(3.3)$ & 163 & 0.37 & $2,650(93.6)$ & $125(4.4)$ & $57(2.0)$ \\
\hline intergenic region & $3,628(4.3)$ & 167 & 0.35 & 3,431 (94.6) & 130 (3.6) & $67(1.8)$ \\
\hline
\end{tabular}

false-positive mutations. The lower mutation burden in our study may also be explained by the identification in our cohort of two samples with an extremely low mutational burden $(<0.2$ mutations/Mbp). Most of the identified mutations were singlenucleotide substitutions (79,960 (94.5\%), predominantly C>T transitions), followed by double substitutions (3,128 (3.7\%), predominantly CC>TT transitions) and short ( $<4 \mathrm{nt})$ indels (1.483 (1.8\%)) (Table $\mathbf{1}$ and Figure 1B). The higher frequency of indels in noncoding regions most likely results from the excess of low complexity sequences, which cause polymerase slippage.

To estimate the fraction of false-positive mutations, we resequenced (with Sanger sequencing) 52 mutations representing different types of alterations, including 39 substitutions and 13 indels (Table S5). The analysis confirmed $51 / 52$ of the mutations, indicating a very low (2\%) fraction of false-positive mutations. The fraction may be even lower, as the only unconfirmed mutation (double substitution CC $>\mathrm{TT}$ in $M Y C N$ ) was present in a low fraction of reads (7\%), which is generally beyond the sensitivity of Sanger sequencing.

\section{Mutational Signatures}

In the next step, we analyzed sample-specific mutational signatures to recognize the mutational processes playing a role in the mutagenesis of the analyzed BCC samples. Shortly, a mutational signature is a frequency pattern for different types of mutations (taking into account direct nucleotide context, -1 and +1 position) characteristic of particular cancer or cancer type. The pattern may reflect a main mutagenic process or a type of DNA repair deficiency that is specific to a given cancer. Originally based on analysis of single nucleotide variants, 30 distinctive mutational signatures were recognized in pancancer (36) but subsequently, the number of specific cancer signatures has been extended taking into account also other types of variants (53). The analysis showed that most of the samples were predominantly associated with signature 7 (average signature contribution $(\mathrm{SC})=0.7$ ) and to a lesser extent with signature 11 (average $\mathrm{SC}=0.2$ ) (Figures $\mathbf{1 C}, \mathbf{D}$ ). Both signatures consist predominantly of $\mathrm{C}>\mathrm{T}$ substitutions but differ in the sequence context of the substitutions. Signature 7 is associated with UV irradiation exposure and commonly occurs in melanoma and head and neck cancer. A hallmark of signature 7 is the frequent occurrence of double $\mathrm{CC}>\mathrm{TT}$ substitutions resulting from UV radiation-induced pyrimidine dimers. Signature 11 was previously found in melanoma and glioblastoma multiforme, often in patients treated with the alkylating agent temozolomide, which is also used in BCC therapy. Only one sample (BCC22) showed a stronger association with signature $11(\mathrm{SC}=0.6)$ than signature $7(\mathrm{SC}=$ 0.3 ). None of the analyzed samples showed an association with signatures 1,2,5, and 13, which are frequent in most cancer types. This may indicate that the deamination of 5-methylcytosine (5meC) predominantly induced by AID/APOBEC cytidine deaminases (attributed to the abovementioned signatures) does not play a role in the pathogenesis of BCC.

The comparison of the nodular and superficial BCC samples showed no substantial difference in terms of mutation burden or mutation types, with the exception of the contribution to mutational signature 7 , which was higher for the nodular than superficial samples (Figure 1E), consistent with the higher UV radiation exposure of nodular BCCs.

\section{Hotspot Mutations}

As recurrent mutations may be indicators of the cancer-related function of the mutated genes, we first looked for hotspots defined as genomic positions mutated in at least 3 samples ( $>10 \%$ of the cohort). In total, we identified 43 hotspots, including 23 hotspots in coding and 20 hotspots in noncoding regions (8 in 5'UTRs, 1 in 3'UTRs, and 11 in introns) (Table S6). Of the coding hotspots, 16 resulted in missense mutations, and 7 were synonymous substitutions. As the majority of synonymous mutations result from randomly occurring neutral alterations, we did not analyze the synonymous hotspot further. Although it has to be noted that the functionality of individual synonymous mutations cannot be unequivocally ruled out $(51,54,55)$. For example, $315(\sim 2.1 \%)$ of the detected in our study synonymous mutations were predicted to be exonic splice-site mutations. Also, synonymous mutations located inside exons may affect different regulatory elements including exonic splicing enhancers and silencers (55). As shown in Table S6, some of the hotspots were located in genes annotated in the COSMIC Cancer Gene Census (CGC) database and/or in genes playing a role in cancer or skin function.

\section{Hotspot Mutations in Coding Regions}

Of the coding mutations (Table S6), the most commonly identified in our study (in 5 samples) was the c.1292C>T (Ser431Phe) substitution, located at chr14:103,131,144 in the Sec6 domain of TNFAIP2, which encodes a multifunctional protein playing a role in angiogenesis, inflammation, cell migration and invasion, cytoskeleton remodeling, and cell membrane protrusion formation (56-59). Nonetheless, 
A

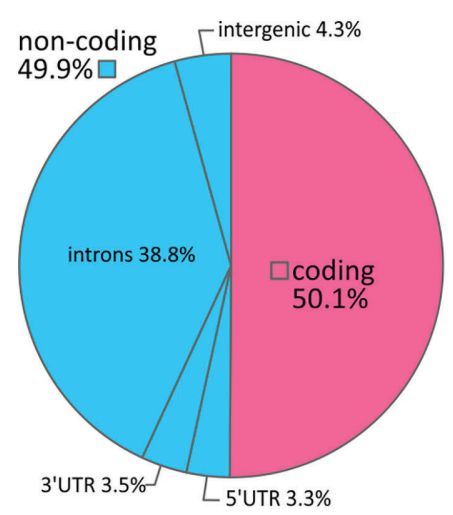

B

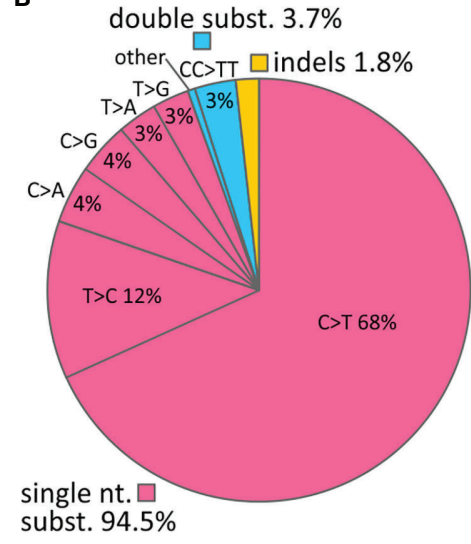

C

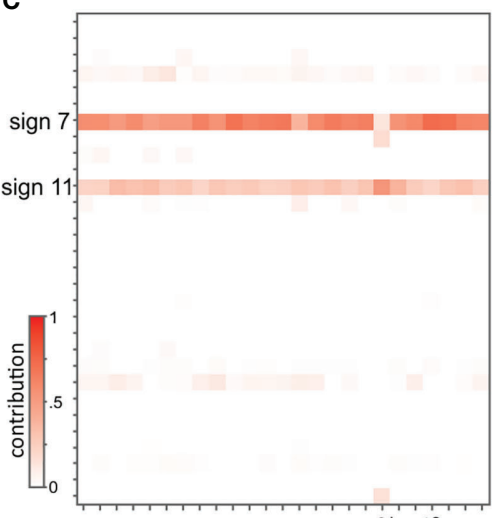

BCC samples

D
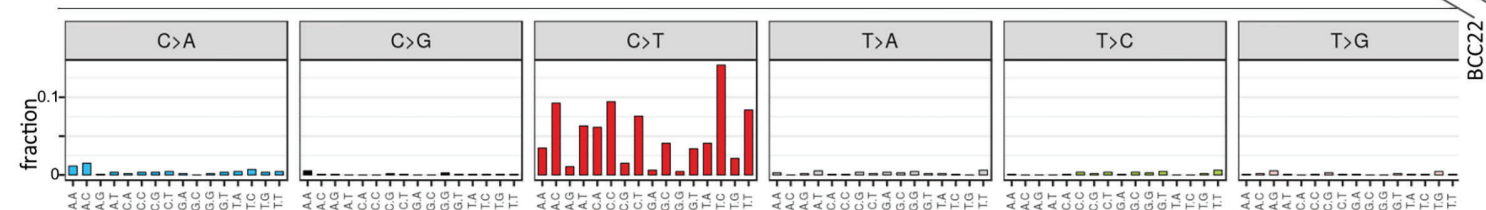
nucleotide context
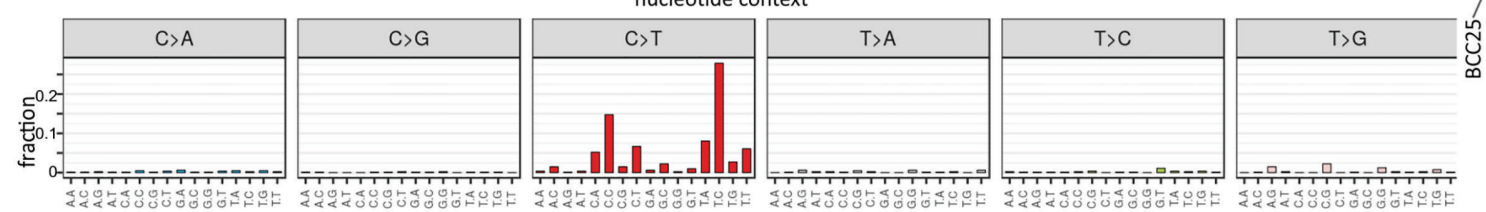
nucleotide context

E
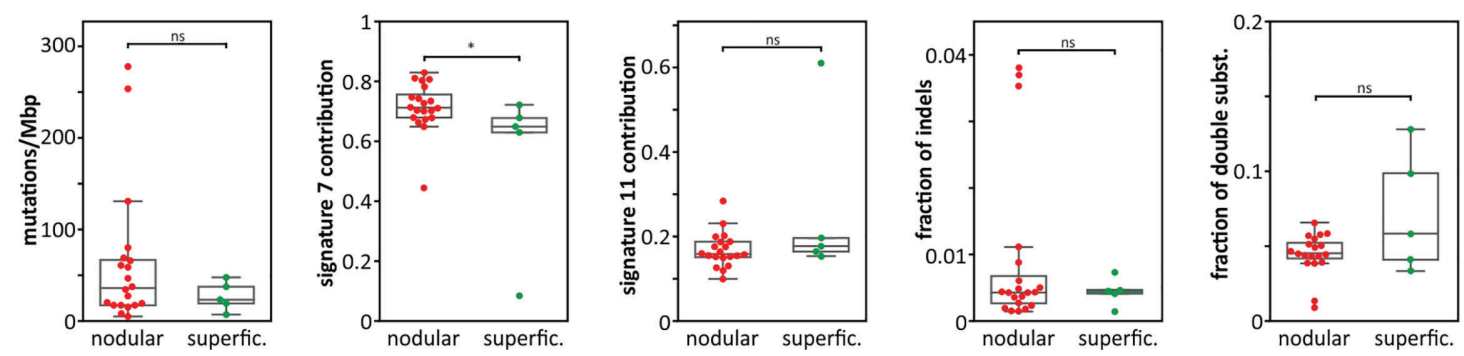

FIGURE 1 | Mutation distribution, mutational signatures, and comparison of superficial and nodular BCC subtypes. (A) Frequency of mutations in particular gene/genomic regions. (B) Frequency of mutation types. (C) Heatmap showing the contribution of the mutational signatures (rows) to the analyzed BCC samples (columns). Higher color intensity indicates a higher contribution (as indicated on the scale bar). (D) Representative mutation distribution plots of samples with a high association with signature 7 (sample BCC25) and signature 11 (sample BCC22). (E) Comparison of nodular and superficial BCC samples in terms of (from the left) mutational load, signature 7 and

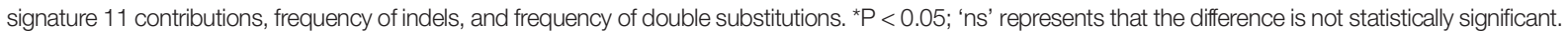

TNFAIP2 is not well-recognized in cancer, and the hotspot or other mutations in the gene have not been reported before. Another coding hotspot, mutated in 3 samples with the c.655C $>$ T (Pro219Ser) substitution, was located at chr7:148,827,237 in EZH2; EZH2 encodes an essential subunit (methyltransferase) of polycomb repressive complex 2 (PRC2), which plays a role in histone methylation and gene silencing (60). EZH2 is a well-known oncogene associated with a more aggressive form and poorer prognosis of many cancers, including melanoma, squamous cell carcinoma (SCC), and BCC, with demonstrated increased expression in SCC [compared to normal skin and SCC precursor actinic keratosis (AK)] (61) and aggressive BCC (62). Both gain- and loss-of-function mutations in EZH2 have often been found in myeloid leukemias and lymphomas but are not common in solid tumors. Contrary to the previously detected mutations clustering mostly in the catalytic SET domain $(63,64)$, the hotspot detected here was located in the N-terminal (NT) part 
of the protein, which, among other areas, is responsible for interaction with histones (65). Whether the mutations may affect the interaction warrants further investigation. To the best of our knowledge, this mutation hotspot has not been observed in any cancer, including BCC.

An additional interesting coding hotspot (mutated in 3 samples) was located at chr15:40,382,906-40,382,907. The hotspot was mutated with either the $c .71 \mathrm{C}>\mathrm{T}$ substitution or the c.71_72delinsTT double substitution (note that double substitutions are annotated as deletion/insertion (delins) variants according to HGVS nomenclature), both resulting in the Ser24Phe missense mutation affecting the NT part of the KNSTRN protein [also known as small kinetochore-associated protein (SKAP)], which plays a role in maintaining chromatid cohesion and proper chromatid separation during anaphase (66). KNSTRN mutations (predominately the Ser24Phe hotspot mutation) were first detected in 19\% of SCCs and 13\% of AKs (67). Subsequent analysis of The Cancer Genome Atlas (TCGA) datasets showed that the KNSTRN mutations also occur in 5\% of melanoma samples but are rare in other cancers. Later, KNSTRN mutations were also identified in $2 \%(15)$ and $10 \%$ (68) of BCCs. These findings together with this study confirm that KNSTRN mutations are specific to UV radiation-related skin cancers. Consistent with the role of KNSTRN, it was shown that KNSTRN mutations in SCC affect proper chromosome separation and are associated with increased chromosome instability, expressed as a fraction of the genome with copy number alterations (CNAs) (67). Although there was a similar number of tested samples, the association of the KNSTRN mutations with CNAs was not confirmed in BCC, neither in a study by Jaju et al. (68) nor in our study (Figure S1). It is worth noting that it was also shown that KNSTRN plays a role in UV radiation-induced apoptosis (69); however, the effect of the mutations on avoidance of apoptosis by BCC cells or any other cancer cells has not yet been tested.

\section{Hotspot Mutations in Noncoding Regions}

The most frequently mutated hotspot of all the hotspots detected in the study (mutated in 8 samples) was located at chr11:64,270,066$64,270,067$ in the 3'UTR of BAD and has never been reported before. The hotspot encompasses 4 different substitutions (c. ${ }^{\star} 142 \mathrm{C}>\mathrm{A}, \quad$ c. ${ }^{\star} 142 \mathrm{C}>\mathrm{T}, \quad c^{*}{ }^{\star} 142{ }_{-}{ }^{\star} 143$ delins TT and $c .{ }^{\star} 143 \mathrm{C}>\mathrm{T}$; Table S6 and Figure 2A), located 142 or 143 nucleotides (nt) downstream of the stop codon. The protein encoded by the gene is a member of the BCL-2 family, which plays a role in the positive regulation of cell apoptosis. The gene is commonly implicated in many cancers $(70,71)$; however, to the best of our knowledge, this hotspot has not been reported before in any cancer.

Next, another novel noncoding hotspot mutated in 5 samples located at chr16:72,008,760-72,008,761 in the 5'UTR of DHODH was identified. The hotspot encompasses two different substitutions, c. $-5 \mathrm{G}>\mathrm{A}$ and c.-5_-4delinsAA, affecting the Kozak sequence (Table S6 and Figure 2B). DHODH is not well studied in cancer, but it has recently been demonstrated that it plays an important role in the carcinogenesis of SCC and other UV radiation-induced skin cancers $(72,73)$.

Another mutated noncoding hotspot from our study worth mentioning was found in 4 samples with the c.-77C>T substitution and was located at chr7:56,106,490 in the 5'UTR of CHCHD2, also known as MNRR1 (Table S6 and Figure 2C). The analysis of the entire CHCHD2 5'UTR showed one more recurrent (in 2 samples) substitution, c. $-134 \mathrm{G}>\mathrm{A}$, located at chr7:56,106,547, resulting in a total of 6 mutations in the 5 'UTR in 6 samples. Interestingly, frequent mutations in the hotspot in the 5'UTR of CHCHD2 were previously reported in melanoma (74).

Finally, we identified a hotspot located at chr1:153,990,763 in the 5'UTR of RPS27 (encoding a ribosomal protein component of the $40 \mathrm{~S}$ subunit) that was mutated in 3 samples with the c.$34 \mathrm{C}>\mathrm{T}$ substitution. Mutations in the promoter/5'UTR of RPS27 (including the hotspot mutation) have been identified before in $\sim 10 \%$ of melanoma samples $(74,75)$ but have never been reported in BCC or other skin cancers. Subsequent in vitro functional studies showed that the RPS27 5'UTR hotspot mutation decreases RPS27 mRNA levels and that decreased levels of RPS27 are associated with a worse prognosis of melanoma patients and drug (vemurafenib and palbociclib) sensitivity of melanoma cells (76).

\section{Computational Analysis of the Identified Noncoding Hotspots and Comparison With External Datasets}

To further characterize three noncoding hotspot mutations, two not previously reported in $B A D$ and $D H O D H$ and one in CHCHD2 previously reported in melanoma (74), we analyzed their potential impact with a number of computational tools and investigated their incidence in other cancers using external datasets of a large cohort (>10,000 samples) of TCGA samples, representing 33 different human cancer types (including 469 skin cutaneous melanoma (SKCM) samples but not including BCC or SCC samples). Note that the list and the standard abbreviations of all TCGA cancer types are in Table S3.

In total, in the TCGA samples, we identified 28 mutations in the BAD 3'UTR (Figure $\mathbf{2 A}$ ). The mutations were found predominantly in SKCM samples (15 mutations in 12 (2.6\%) SKCM samples), including 4 mutations in the hotspot (residues c. ${ }^{\star} 142 \mathrm{C}$ and c. $^{\star} 143 \mathrm{C}$ ) identified in BCC, and 6 c. ${ }^{\star} 77 \mathrm{C}>\mathrm{T}$ mutations, constituting an additional hotspot in the 3 'UTR, not occurring in BCC. In other cancers, 3'UTR mutations were very rare (Figure $\mathbf{2 A}$ ). In contrast with the mutation frequency in the 3'UTR, mutations in other parts of the gene, including the coding region ( $n=26$, predominantly missense or synonymous), were rare (not exceeding $1 \%$ in any cancer) and randomly distributed between different cancer types (excluding SKCM). The exclusiveness of the SKCM and BCC mutations in the 3'UTR vs. other parts of the gene (enrichment compared to other cancer types; Fisher's exact test; $\mathrm{p}<0.0001$ and $\mathrm{p}=0.0005$, respectively) precludes an accidental occurrence of the mutations, solely as a result of some region- and/or mutagenesis-related mechanisms and argues for the cancerdriven selection of the 3'UTR mutations in BCC and SKCM (and likely also in other UV irradiation-related cancers).

Next, with the use of TargetScan, we identified 3 miRNAs (miR-7515, miR-3202, and miR-6125) whose predicted targets (seed-interacting sequences) were disrupted by hotspot mutations (Figure 2A). However, as (i) none of these targets 
A

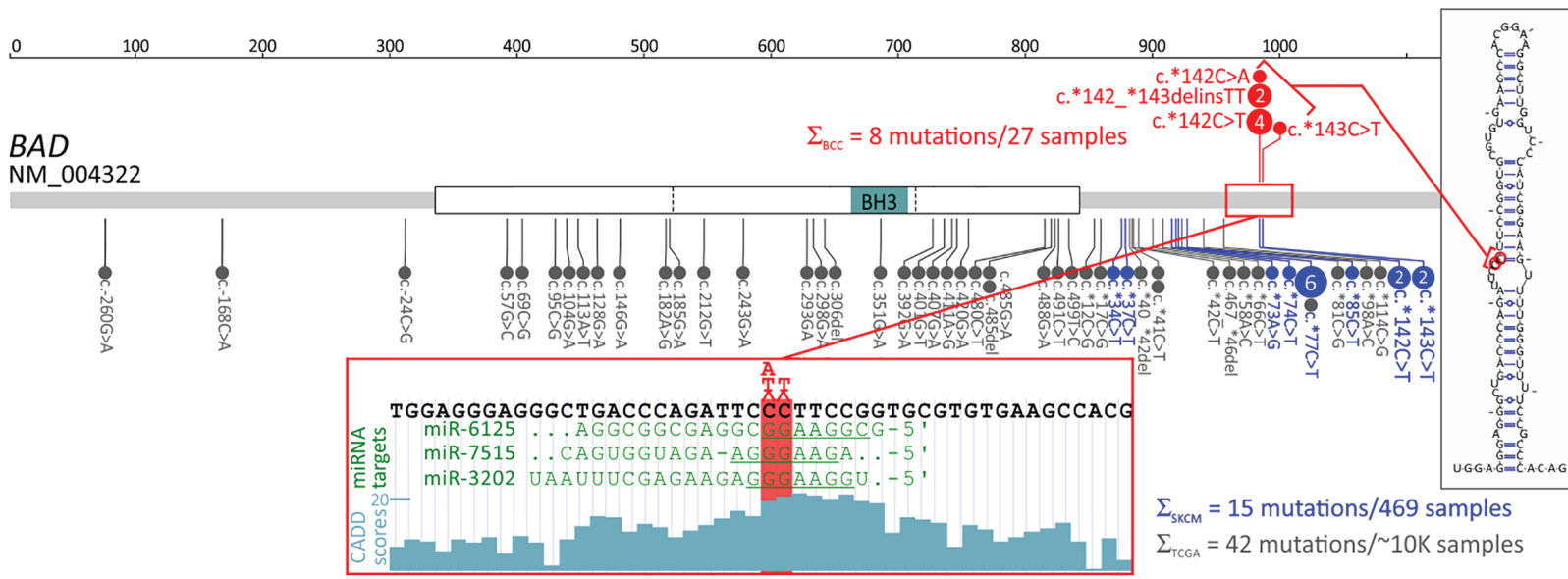

B

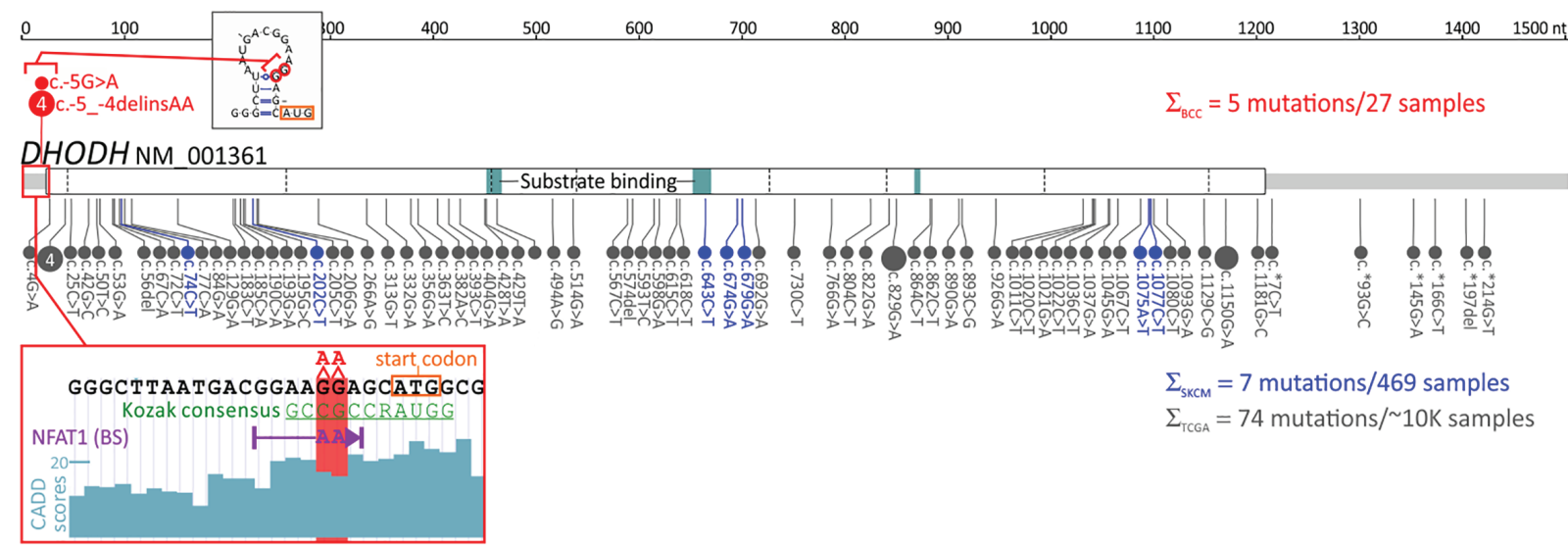

C

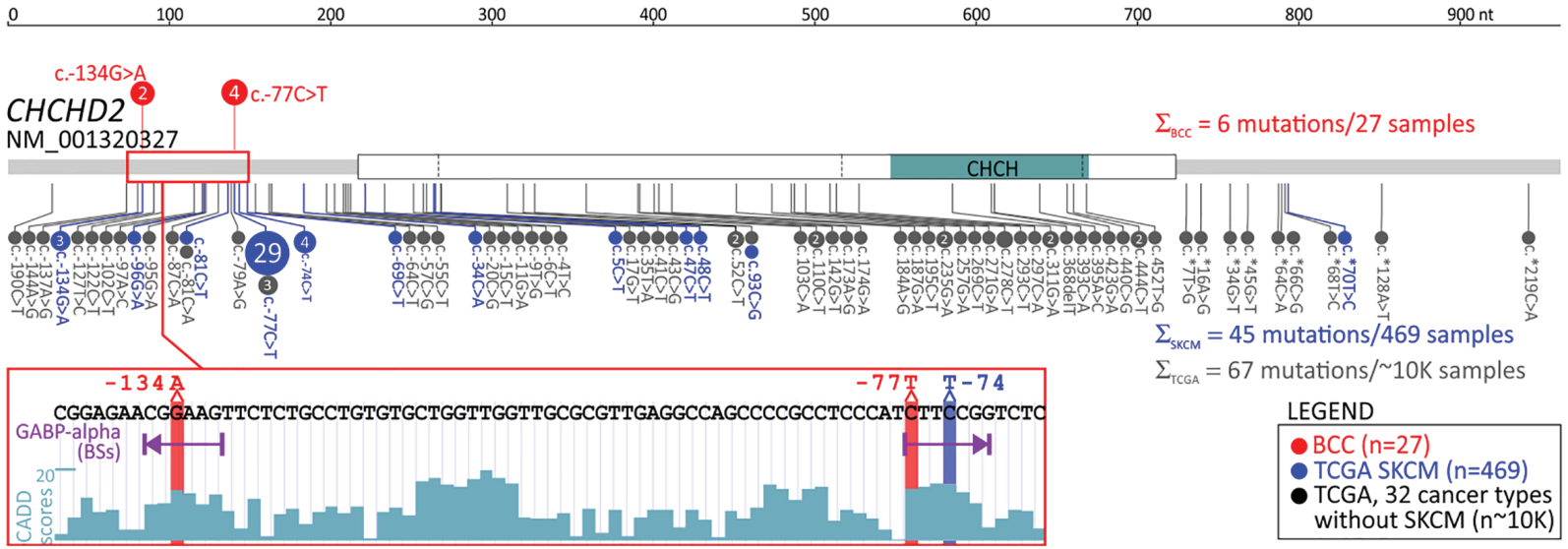

FIGURE 2 | Distribution of mutations in the selected genes with the identified mutation hotspots in noncoding areas. (A-C) Maps of the $B A D, D H O D H$, and CHCHD2 genes, with the exon structure and protein functional domains indicated. Mutations are visualized in the form of lollipop plots along with the gene maps, and the size of a mutation symbol (circle) is proportional to the number of mutations. Mutations identified in BCC (red) are shown above and mutations identified in SKCM (blue) and other TCGA cancers (gray) are shown below the maps. The inset below each map shows the detailed sequence context of the hotspot mutations, along with CADD score graphs, indicating the functional relevance of particular positions and other sequence characteristics (i.e., (in A) predicted miRNA target sites, (in B) the Kozak consensus sequence and NFAT1 transcription factor binding sites (BSs) created by the hotspot mutation, and (in C) the GABP-alpha transcription factor BSs disrupted by the hotspot mutations). The additional insets in (A, B) show computationally predicted RNA secondary structures generated from RNA sequences directly flanking the hotspots. * represents stop codon. 
has been validated by any means [miRTarBase (77)], (ii) none of these miRNAs have been confidently validated (via miRBase or miRGeneDB), and (iii) none of these miRNAs have been found to have expression levels detectable/confirmed in any of the TCGA cancers, it is very unlikely that any of the identified targets are functional. Additionally, the occurrence of SKCM mutations in different positions across the BAD 3'UTR argues against the possibility that the driving force of the mutations is a disruption of a particular miRNA target. Some clue for the functionality of the BCC hotspot may be its location in the 5 ' arm of the $\sim 40 \mathrm{bp}$ long stable hairpin RNA structure motif $(\mathrm{dG}=-39.6 \mathrm{Kcal} / \mathrm{mol})$, which is destabilized (by $\sim 2 \mathrm{Kcal} / \mathrm{mol}$ ) by the hotspot mutations (Figure 2A).

The analysis of TCGA data showed no mutation in the BCC hotspot or any other mutation in the $\mathrm{DHODH} 5$ 'UTR in any of the TCGA cancer types, even though different mutations $(n=81)$ were identified in other parts of the gene, including 75 mutations in the coding region (Figure 2B). The other mutations, however, were randomly distributed along the gene sequence and between different cancer types, and only two of the coding mutations were deleterious (frameshift) mutations. This result indicates that the DHODH 5'UTR hotspot mutations are BCC-specific mutations, and the absence of these mutations in other UV radiation-related cancers makes it unlikely that the frequent occurrence of the mutations in BCC is solely due to a random effect of UV irradiation. The 5'UTR of DHODH is very short (21 bp). Although hotspot mutations occurred in the Kozak sequence, which is important for the initiation of translation, neither wildtype nor mutant alleles affected the consensus Kozak sequence nucleotides (at positions -4 and -5); therefore, the ATGpr (78)), and NetStart 1.0 (GedersenAG (79) tools predicted the mutations to have a minor effect on the effectiveness of translation under standard conditions. However, this result does not exclude an effect of the mutations under specific conditions, such as hypoxia, UV exposure, or cancer.

The analysis of RNA secondary structure showed that the hotspot mutations slightly modified (decreased the stability of) a small hairpin motif predicted to be formed by an RNA sequence directly flanking the hotspot (Figure 2B). The mutation may also destabilize the potential long-range interaction of the sequence flanking the mutations with the sequence located $200 \mathrm{nt}$ downstream. Analysis of the 5'UTR sequence (80) showed that the double substitution (GG>AA) at the hotspot creates a consensus binding site for the NFAT1 transcription factor (Figure 2B), which is expressed in many tissues, including sun-exposed and non-sun-exposed skin (GTExPortal; GTEx Consortium Science 2020), and implicated in many cancers, including melanoma $(81,82)$.

In total, in TCGA data, we identified 63 mutations in the CHCHD2 5'UTR (Figure 2C). The mutations were found predominantly in SKCM samples (40 mutations in 39 (8.5\%) samples), including 29 c. $-77 \mathrm{C}>\mathrm{T}$ mutations and 3 c.-134G $>\mathrm{A}$ mutations, located in the hotspot positions identified in BCC. Additionally, we identified 4 samples with the c.-74C>T mutation, constituting an additional hotspot in the 5'UTR. Only 5 SKCM mutations were located outside the 5'UTR, 4 in the CDS (2 missense and 2 synonymous), and 1 in the 3'UTR (one mutation) (Figure 2C). In other cancers, there were rare 5'UTR mutations, including 4 mutations in HNSC and UCEC, 3 mutations in BRCA, and 12 mutations in other cancers. Three of these mutations coincided with the c.-77 hotspot. The positions of BCC/SKCM hotspot mutations seem to be nonrandom because they were all located in and all disrupted two distinct GABP-alpha transcription factor binding sites [mapped with the use of MotifMap (50)] (Figure 2C).

\section{Frequently Mutated Genes}

Next, we looked at the overall frequency of mutations in the genes, separately analyzing mutations in coding regions, 5 'UTRs, 3'UTRs, and introns (defined in Materials and Methods; listed in Table S7). Although they were not considered frequently mutated, in this section, we also report genes with any mutations in a coding region if they were detected in a pathway of a recurrently mutated gene. In the analysis of frequently mutated regions, we focused mostly on genes functionally related to cancer (annotated with CGC and a manual literature search) and genes playing a role in skin function.

\section{Genes Frequently Mutated in Coding Regions}

In total, we identified 606 genes frequently mutated in coding regions. The most frequently mutated was $P T C H 1$, with a total of 24 mutations in 20 BCC samples, including 5 missense, 4 splicesite, and 15 deleterious (nonsense or frameshift) mutations (Figure 3A). Mutation c.3450-1G>A located upstream of exon 21 was one of the splice-site mutations and was also observed in another study (14), which suggests its recurrence in BCC. We tested and confirmed the exon-skipping effect of the mutation with the use of exon-junction PCR and Sanger sequencing analysis (Figure 3A). The other genes from the hedgehog pathway recurrently mutated in our cohort were GLI2, which was mutated in 5 samples, and SMO, which was mutated in 4 samples (Figure S4 and Figure 4). The combined frequency of $S M O$ and GLI2 mutations was much lower in samples with (4/20; $20 \%)$ than in those without $(4 / 7 ; 57 \%)$ PTCH1 mutations, which suggests mutual exclusiveness of these mutations (Figure 4). Altogether, 24 (88\%) samples had mutations in genes involved in the hedgehog pathway. Other frequently mutated cancer-related genes were TP53 (7 missense, 8 deleterious, and one splice-site mutation in 13 samples) (Figure 3B); $M Y C N$ (8 missense mutations in 8 samples), NOTCH1 ( 8 missense and 2 deleterious mutations in 8 samples), NOTCH2 (3 missense, 3 deleterious, and 2 splice-site mutations in 7 samples), $\mathrm{NOTCH} 3$ (6 missense mutations in 5 samples; note that the $\mathrm{NOTCH}$ mutations colocalized with the regions of the loss-of-function mutations identified in other solid tumors, e.g., in SCCs (83), LATS1 (5 missense and one deleterious mutation in 5 samples), and ARIDIA ( 5 missense mutations in 5 samples) (Figure $\mathbf{4}$ and Figure S2). The mutations in the abovementioned genes are generally consistent with mutations observed before in BCC (14, 15). Additionally, we identified very frequent mutations (18 missense and 1 deleterious) in PTPRD (Figure 3C), a tumor 


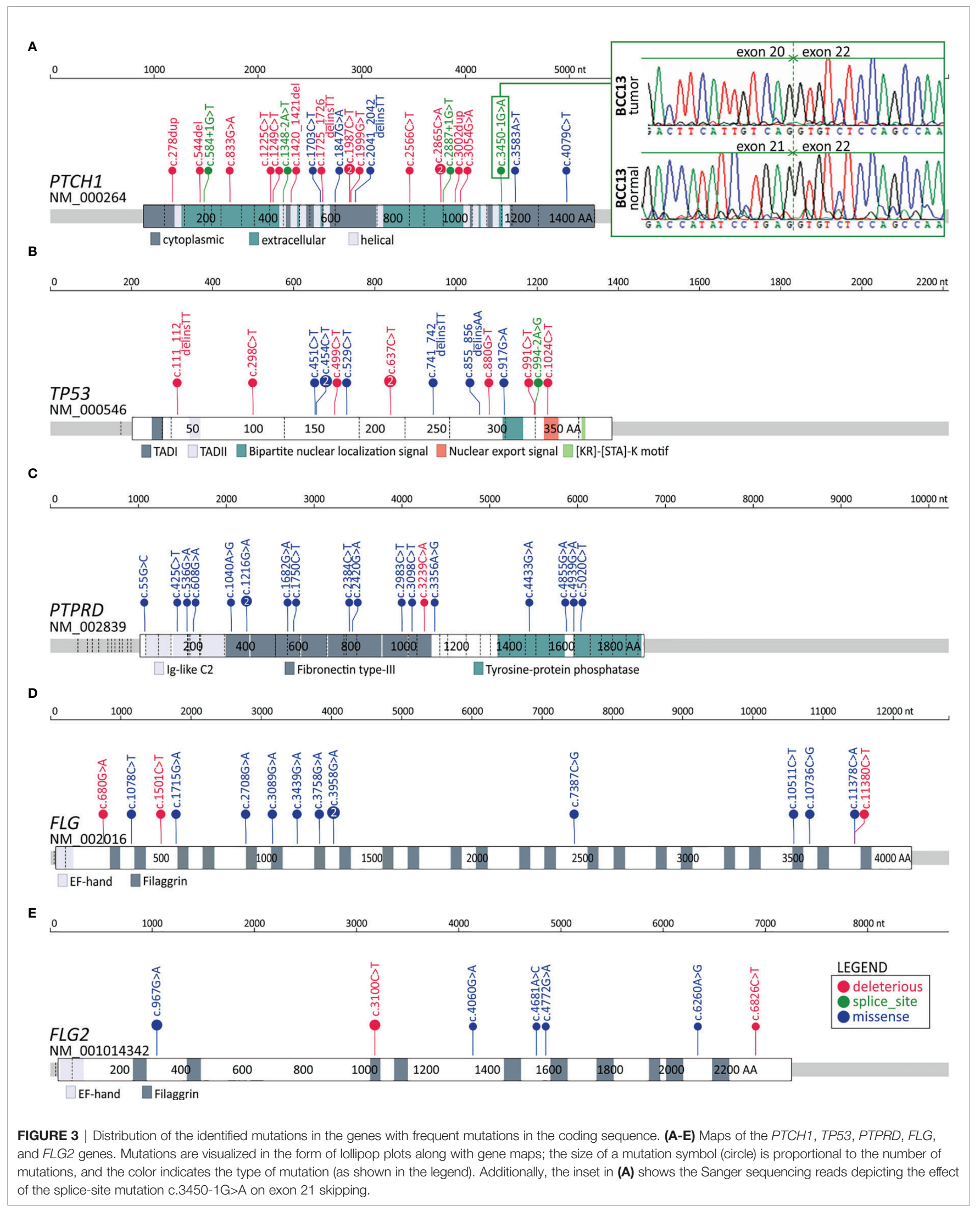




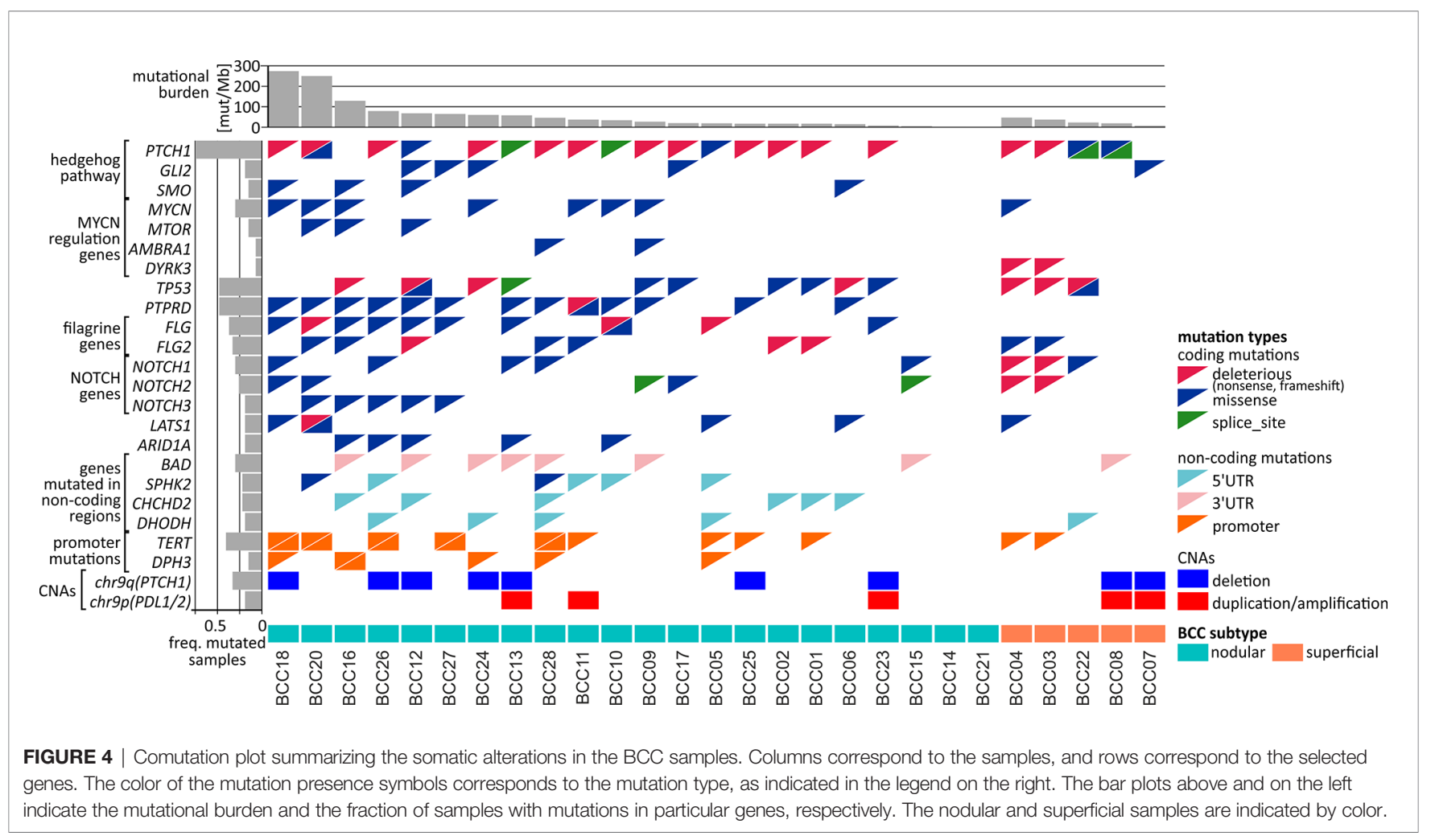

suppressor frequently mutated in many cancers, including melanoma and cutaneous SCC (84-88), in 13 samples, but these have never been reported as frequently mutated in BCC.

Interestingly, in addition to mutations in $M Y C N$, we also noticed recurrent (although not frequent) mutations in three other genes in the MYC/MTOR regulatory network, i.e., MTOR, $D Y R K 3$, and AMBRA1 (Figure 4), which have not been reported as mutated in BCC. The MTOR missense/activating mutations identified in other cancers are considered biomarkers for therapy with mTOR pathway inhibitors (89).

Finally, we found a high frequency of mutations in the FLG (15 mutations in 10 samples) and FLG2 (9 mutations in 9 samples) genes (Figures 3D, E and Figure 4), encoding profilaggrin and filaggrin-like proteins, precursors of filaggrin. Filaggrin is an important component of the stratum corneum of the epidermis that plays a role in maintaining epithelial homeostasis and barrier functions (90) and is a substrate for trans-urocanic acid (UCA) and pyrrolidone carboxylic acid (PCA), which are suggested to serve as a natural UV radiation barrier (91). Although frequent mutations in the FLG/FLG2 genes have been previously observed in other cancers, the mutations were usually considered random (passenger). Here, however, we observed a relatively high proportion of deleterious nonsense mutations, altogether occurring in 6 samples. Additionally, the analysis of the entire cohort of TCGA samples showed that the frequency of the FLG/ FLG2 mutations observed in our study in BCC substantially exceeds the frequencies of the mutations in other cancers, including melanoma (the next most frequently mutated cancer) (Figure S3).

\section{Genes Frequently Mutated in Noncoding Regions}

Among the 11 genes frequently mutated in the 5'UTR (Table S7) there were $\mathrm{DHODH}$ and $\mathrm{CHCHD} 2$ with the hotspot mutations described above (see subsection Hotspot mutations). Of interest may also be SPHK2, with 4 dispersed mutations in 4 samples, whose function as both a proapoptotic gene suppressing cell growth and an oncogene promoting cell proliferation has been proposed (92-96). SPHK2 also had mutations in its coding region (Figure 4).

Among the 11 genes frequently mutated in the 3'UTR (Table S7), in addition to BAD described above (see subsection Hotspot mutations), we also identified 8 mutations in the 3'UTR of SMIM27 (also annotated as lncRNA TOPORS-AS1); the overexpression of SMIM27 was found to be associated with favorable outcomes in breast cancer (97).

Finally, we identified 289 genes (15 annotated in CGC) frequently mutated in introns (Table S7). Interestingly, among the genes was PTCH1, which, in addition to 4 splice-site mutations (mentioned above), also had other 4 intronic mutations (in total, 8 intronic mutations). Other genes with frequent mutations in introns included PTPRD (14 mutations in 9 samples), which also frequently had mutations in the coding region; NOTCH2 (6 mutations, including 2 splice-site mutations in 6 samples), which also frequently had mutations in the coding region; ERBB4 (6 mutations in 6 samples), a well-known oncogene playing a role in many cancers [reviewed in (98)]; and DROSHA (5 mutations in 5 samples), which encodes a core enzyme (nuclease) of the miRNA processing pathway and has been shown to be upregulated in BCC (99). 


\section{Mutations in the TERT and DPH3 Promoters}

The only noncoding mutations previously studied in BCC are mutations recurrently occurring in promoters of TERT and DPH3 $(27,100,101)$. As these promoters were not covered in our exome sequencing experiment, we performed Sanger sequencing for these regions. As a result, we have detected 16 mutations in $11(41 \%)$ patients in the TERT promoter and 6 mutations in 5 (19\%) patients in the DPH3 promoter (Figure 4). All TERT mutations were detected in previously described positions and well-known hotspots responsible for the recruitment of transcription factors activating expression of TERT in cancer, including 2 double substitutions c.-139_138delinsAA, 9 substitutions c.-146G>A, 2 substitutions c.$101 \mathrm{G}>\mathrm{A}$, and 3 other substitutions (c. $-150 \mathrm{G}>\mathrm{A}, \mathrm{c} .-100 \mathrm{G}>\mathrm{A}$, and c. $-99 \mathrm{G}>\mathrm{A})$. Also, DPH3 mutations were located in positions described before $(27,102)$, including 3 double substitutions c.-122_-121delinsTT, and 3 other substitutions (c. $-150 \mathrm{C}>\mathrm{T}$, c. $-122 \mathrm{C}>\mathrm{T}$, and c. $-121 \mathrm{C}>\mathrm{T}$ ).

\section{Driver Genes in BCC (OncodriveFML Analysis)}

To further investigate the mutations/mutated genes, we used OncodriveFML, which allows the prediction of the cancer driver potential of both coding and noncoding regions/genes based on functional mutation (FM) bias (39). As shown in Figures 5A-C and Table S8, we identified 14 potential cancer driver genes based on mutations in coding regions (CDS-drivers), a disproportionately high number of 36 potential cancer driver genes based on mutations in 5'UTRs (5'UTR-drivers), and 7 potential cancer driver genes based on mutations in 3'UTRs (3'UTR-drivers). No potential cancer driver gene was identified based on the mutations in introns.

In addition to 4 CDS-drivers (PTCH1, TP53, TGFB1I1, and $C A R D 6)$ also identified as frequently mutated, it is worth noting $R O R A$, recently shown to play an important role in restraining allergic skin inflammation (103). Other interesting genes were PRDM9 and ZNF281, both of which play a role in DNA repair and have been shown to be responsible for frequent mutations in cancer $(104,105)$. None of these genes were previously implicated or identified as frequently mutated in BCC.

Among the 5'UTR-drivers, 6 were also identified as frequently mutated: DHODH, CHCHD2, and SPHK2 (described above), as well as POLR2M, NPC1, and NELL2. Additionally, it is worth noting $I K B K B$ (mutated in 3 samples but not reported before as mutated in BCC) shown to act as a tumor suppressor in nonmelanoma skin cancers and noncancerous skin lesions; it was also shown that deletions of the gene lead to skin inflammation, hair follicle disruption, hyperplasia, and SCC development (106-109).

Among 3'UTR-drivers, two genes (mentioned above), i.e., $B A D$ (the most significant 3'UTR-driver) and SMIM27 were also identified as frequently mutated. Additionally, it is worth mentioning the transcription factor gene POU3F2 (mutated in 3 samples), that plays a role in the invasiveness and metastasis of melanoma, and is controlled by miR-211 $(110,111)$ and miR-107 (112). Although the mutations were not located in the predicted miR-107 and miR-211 binding sites, they may affect the structure of the 3'UTR and thus indirectly change accessibility to these or other miRNA targets.

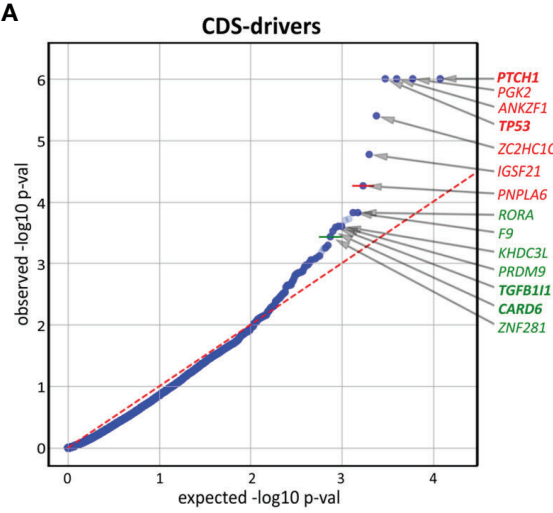

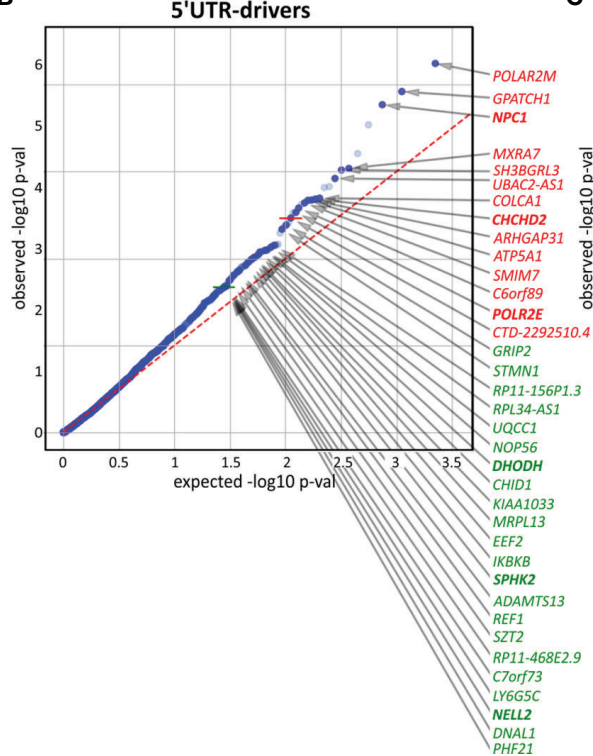

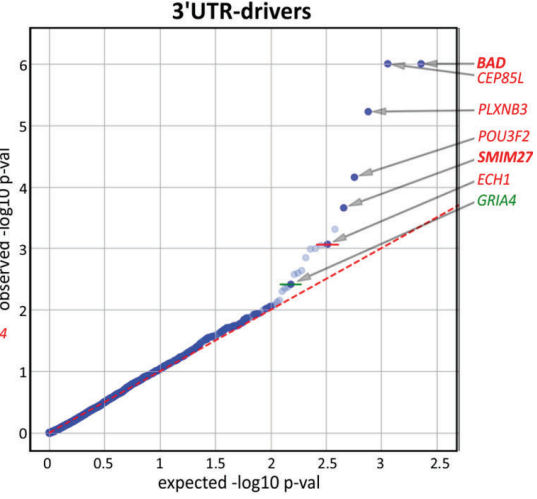

FIGURE 5 | Identification of potential cancer drivers with the use of OncodriveFML. The quantile-quantile (QQ) plots show the distribution of expected ( $\mathrm{X}$-axis) and observed (y-axis) p-values corresponding to FM bias calculated (with CADD score) separately for mutations in (A) coding regions, (B) 5'UTRs, and (C) 3'UTRs. The green and red colors indicate genes defined as significant $(q<0.025)$ and highly significant $(q<0.01)$, respectively, according to OncodriveFML recommendation. 


\section{Analysis of Copy Number Alterations}

As somatic CNAs have not been extensively studied in BCC, in the next step, we performed analysis of both chromosome armlevel and focal CNAs [with GISTIC2 (40)]. At the chromosome arm level, we detected a significant recurring deletion of chr9q ( $\mathrm{q}=1.4 \times 10-6$; occurring in 9 samples), involving PTCH1 (Figures 4, 6), and a significant recurring amplification of chr9p ( $q=0.05$; occurring in 5 samples), involving a region with CD274 (also known as PDL1, encoding PD-L1), CD273 (also known as PDL2, encoding PD-L2), and JAK2 (Figures 4, 6). Although the loss of chr9q has been frequently observed in BCC (reported as loss-of-heterozygosity of PTCH1), gain of chr9p has been reported only in one case of rare metastatic BCC (113). To validate the chromosome 9 CNAs, we developed a multiplex ligation-dependent probe amplification (MLPA) assay with probes covering the entire chromosome 9 but especially focusing on the region containing PTCH1 (chr9q22.32) and the region harboring $P D L 1, P D L 2$, and $J A K 2$ (chr9p24.1) (Figure 6). The MLPA analysis confirmed CNAs in all tested samples as detected by GISTIC2, and examples are shown in Figure 6.

CNA analysis also showed 54 regions of significant focal deletions, including 27 regions containing skin/cancer-related genes, and 56 significant amplifications, including 20 encompassing skin/cancer-related genes (Figure 6 and Table S9). The elements involved in the most significant focal deletions were CDK11A (chr1p36.33; q=2.4x10-5; occurring in 6 samples), whose loss induces skin carcinogenesis (114); the LCE cluster (chr1q21.3; $\mathrm{q}=2.4 \times 10-6$; occurring in 4 samples), including genes such as LCE2 and $L C E 3$, which play a role in maintaining skin barrier function and whose deletion has been associated with psoriasis (115); and the HLA-D cluster (HLA-DP, $-D Q$, and $-D R$, chr6p21.32; $\mathrm{q}=2 \mathrm{x} 10$ 4 ; occurring in 3 samples), encoding components of major
A

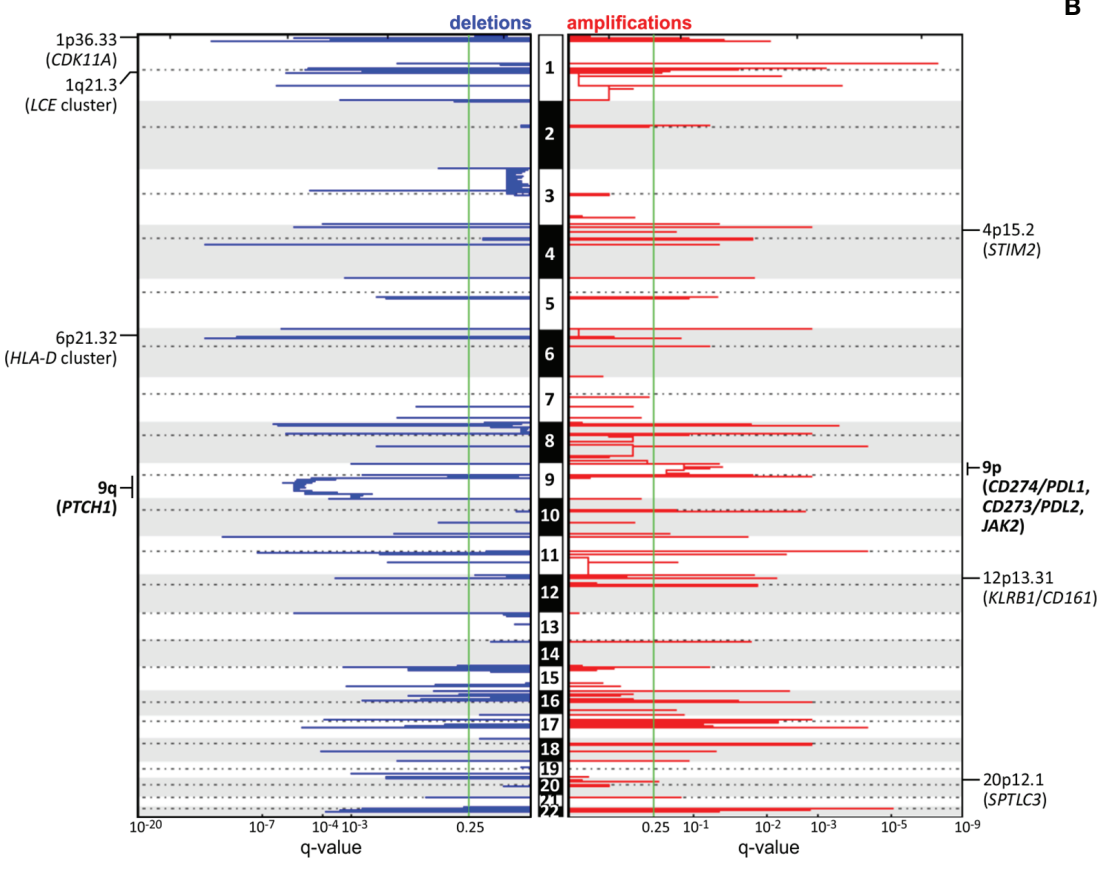

B
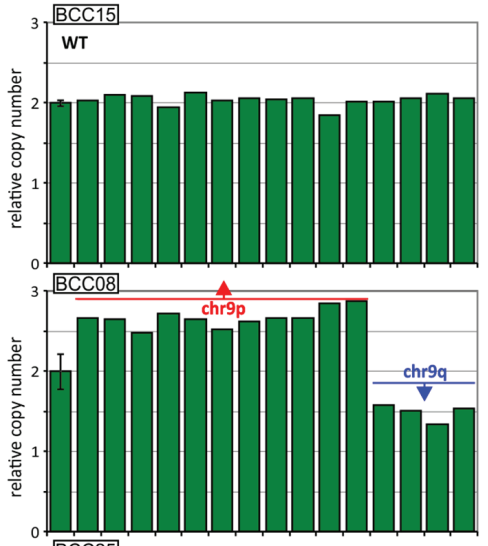

$$
\text { BCC25 }
$$

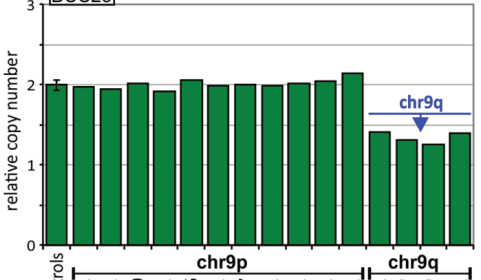

chr9p chr9q

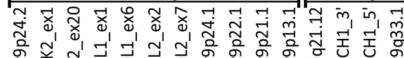

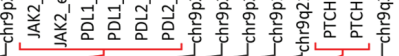

C

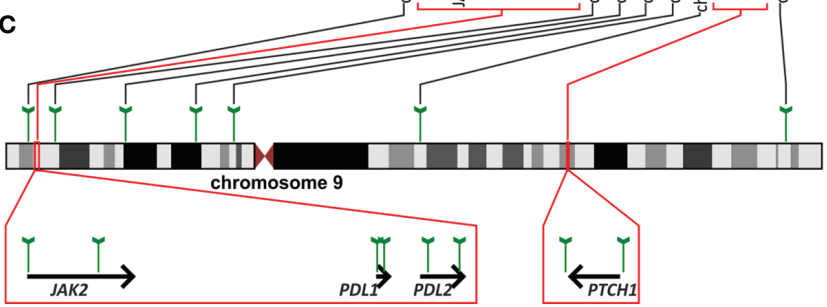

FIGURE 6 | CNA analysis of the BCC samples. (A) GISTIC-estimated q-values for deletions (left, blue) and amplifications (right, red) are plotted along with chromosome positions (vertically). The green line indicates the recommended significance threshold, $\mathrm{q}=0.25$. The selected significantly deleted and amplified regions/ genes are indicated on the graphs. (B) Representative MLPA results (bar plots), showing samples with chromosome 9 CNAs, i.e., chr9q deletion and chr9p amplification, vs. a sample (at the top) with the wild-type (WT) copy number genotype. Each bar plot depicts relative copy number values (y-axis) of the probes specific for regions along chromosome 9 and an average (with standard deviation error bar) signal of control probes (x-axis). (C) Schematic depictions of the localization of the probes on chromosome 9 and in genes of interest. 
histocompatibility complex (MHC) class II molecules, whose increased expression has been associated with increased cancer immunogenicity and better prognosis in BCC, SCC and melanoma (116-122). The skin/cancer-related genes in the most significant focally amplified regions worth mentioning are STIM2 (chr4p15.2; $\mathrm{q}=0.16$; occurring in 2 samples) (123), KLRB1/CD161 (chr12p13.31; q=0.007; occurring in 2 samples) (124, 125), and SPTLC3 (chr20p12.1 q=0.23; occurring in 2 samples) (126).

\section{DISCUSSION}

In this study, we detected thousands of mutations in BCC samples, many of which were clustered in specific genes/ regions or hotspots located in both coding and noncoding regions. Despite the small size of our dataset, our results are in line with those of previous genomic analyses of coding mutations in BCC $(14,15)$, which confirms the reliability of our study. We believe that our results may give valuable insights related to general characteristics of mutations such as mutational burden or mutational signatures and in terms of genes identified as recurrently mutated in coding regions.

Moreover, we extended our analysis to noncoding parts of the genes, which altogether were responsible for $\sim 50 \%$ of the mutations identified by the standard WES approach. Variants in such areas have usually been ignored in previous BCC genetic studies. Many of the identified noncoding hotspots were located in sequences of genes functionally related to cancer or more specifically to UV radiation-related skin cancers. Some of them were reported before in melanoma or identified by us in melanoma TCGA samples, the cancer type most intensively studied in terms of mutations in noncoding regions $(127,128)$. Below, we briefly describe the cancer-related role of the three most interesting genes with hotspot mutations in noncoding regions, i.e., $B A D, D H O D H$, and $C H C H D 2$. Interestingly, all these genes have functions related to mitochondrial activity.

Of all the hotspots detected in our study, the most frequently mutated was the hotspot located in the 3'UTR of BAD. This hotspot had several different mutations affecting 2 nucleotide positions (142 and $143 \mathrm{nt}$ downstream of the stop codon). Due to these mutations, $B A D$ was also classified as being highly mutated in the 3'UTR and as the top most significant potential cancer driver. Consistently, the hotspot and several other positions in the 3'UTR are frequently mutated in melanoma but not in other cancers. BAD belongs to the BCL-2 family, consisting of both proapoptotic and antiapoptotic proteins. It promotes cell death by inducing mitochondrial outer membrane permeabilization (MOMP), allowing the release of cytochrome $c$, and by antagonizing (dimerizing with) antiapoptotic BCL-2 proteins $(129,130)$. On the other hand, phosphorylated BAD may also have antiapoptotic properties, e.g., promoting the survival of melanocytes $(131,132)$. Other functions of BAD include regulation of mitochondrial metabolism (regulation of voltagedependent anion channels and metabolite passage through the outer mitochondrial membrane) and dynamics (regulation of shape changes) (133-139). Although BAD has not been previously implicated in skin cancers, loss or downregulation of other proapoptotic members of the BCL-2 family, i.e., BAX and PUMA, has been shown to promote the development of BCC, SCC, and cutaneous melanoma $(140,141)$. Therefore, a similar effect may be induced by mutations causing more efficient downregulation of $\mathrm{BAD}$.

CHCHD2 is a gene with frequent mutations in the 5'UTR, the hotspot mutation c.-77C $>\mathrm{T}$ and the recurrent mutation c.$134 \mathrm{G}>\mathrm{A}$ (77 and 134 upstream of the start codon). Based on the 5'UTR mutations, $\mathrm{CHCHD} 2$ was classified as a high-priority cancer driver. We showed that the CHCHD2 5'UTR (predominantly the hotspot position) was also frequently mutated (8\%) in the SKCM TCGA samples, which also showed the additional recurrent mutation c. $-74 \mathrm{C}>\mathrm{T}$. The 5 'UTR mutations were also found in whole-genome sequenced Australian melanoma samples (74). The role of the gene has not been intensively studied in cancer, but it was shown that under hypoxic conditions, CHCHD2 is translocated from the mitochondrial intermembrane space to the nucleus, where it binds an oxygen-responsive element in the promoter of cytochrome oxidase $4 I 2$ (COX4I2), encoding a subunit of complex IV of the electron transport chain, and increases its expression. Consequently, $\mathrm{CHCHD} 2$ knockdown downregulates COX4I2 and decreases cell oxygen consumption (142). It was also shown that $\mathrm{CHCHD} 2$ is a negative regulator of mitochondriamediated apoptosis (143). Liu et al. showed that CHCHD2 interacts with antiapoptotic BCL-XL (from the BCL-2 family), which leads to inhibition of proapoptotic BAX and consequently decreases MOMP and apoptosis. In addition, it was shown that CHCHD2 dysregulates multiple genes that play a role in cell migration and cancer metastasis and that its expression is higher in cell lines derived from more aggressive breast tumors (144). Consistent with the function of $\mathrm{CHCHD} 2$ related to mitochondrial metabolism, we found that all BCC/SKCM hotspot/recurrent mutations coincided with and impaired two distinct binding sites of GABP-alpha. As GABP-alpha is known to be a transcription factor involved in the regulation of cellular energy metabolism and cell cycle regulation (145), this finding might hint at a functional role of the mutations in cancer. Of note, germline missense mutations in CHCHD2 are associated with autosomal dominant Parkinson's disease (146).

$\mathrm{DHODH}$ is a gene that showed frequent mutations in the Kozak sequence of the 5'UTR, with hotspot mutations encompassing two different substitutions, c.-5G $>$ A and c.-5_4delinsAA (4 and $5 \mathrm{nt}$ upstream of the start codon). Based on the identified mutations, $D H O D H$ was classified as a candidate cancer driver. The analysis of the entire TCGA cohort $(\sim 10 \mathrm{~K}$ samples from 33 cancer types) showed that no other cancer had mutations in the hotspot or the 5'UTR, indicating that the mutations were BCC-specific. Although DHODH 5'UTR mutations have never been reported before in any cancer, it was shown very recently that $D H O D H$ plays a key role in the carcinogenesis of SCC and other UV radiation-induced skin cancers and facilitates the development of precancerous skin lesions $(72,73)$. Hosseini et al. showed that the $\mathrm{DHODH}$ protein level and enzymatic activity are markedly upregulated in irradiated skin and that an increased level of $\mathrm{DHODH}$ sensitizes the skin to UV irradiation-induced damage. It was 
also shown that DHODH is upregulated in melanoma, in which DHODH inhibition leads to a marked decrease in tumor growth both in vitro and in mouse xenograft studies (147). DHODH inactivation inhibits cell proliferation and induces cell cycle arrest at the $\mathrm{S}$ phase in BCL-2 (pro-apoptotic) deficient melanoma cells (148). DHODH is embedded in the inner mitochondrial membrane, and its canonical role is in the oxidation of dihydroorotate to orotate, an important step in de novo pyrimidine synthesis (which is important in replication and DNA repair). However, a side product of the pathway, ubiquinol $(\mathrm{QH} 2)$, is a source of electrons in the electron transport chain, and DHODH also plays a role in alternative (glucoseindependent) respiration (utilizing amino acids as an energy source) $(72,73,148)$, facilitating cancer development in hypoxic conditions. In addition, it was found that in esophageal SCC, elevated DHODH levels promote cell proliferation by stabilizing $\beta$-catenin (149). The functional effects of the mutations may result from alteration of the Kozak sequence but also the creation of an NFAT1 transcription factor binding site, which is not present in the wild-type sequence. NFAT1 is a widely distributed isoform of the NFAT family of transcription factors and is expressed in tumor cells and the tumor microenvironment (150). The constitutive activation and overexpression of NFAT1 in many cancer types promote the transcription of genes that are crucial for cancer development and progression, including COX2, MMP7, MMP9, and MDM2 (151, 152).

It is worth noting that the only noncoding mutations analyzed in BCC before are the mutations in promoters of TERT and DPH3 (27, 100, 101); which are known to be mutated in many cancers, including melanoma (127, 128). Although our WES design generally did not cover promoter regions, with the use of Sanger sequencing, we confirmed high frequency and high recurrence of promoter mutations in TERT (41\% of patients) and DPH3 (19\%).

Additionally, the whole-genome CNA analysis allowed us to detect two highly significant chromosome-level CNAs. In addition to the expected deletion of chr9q, consistent with the loss of heterozygosity of $\mathrm{PTCH} 1$, we also detected frequent duplication/amplification of chr9p, encompassing the PDL1 and PDL2 genes (which encode the two immune checkpoint proteins PD-L1 and PD-L2, the overexpression of which enables cancer cells to evade the host immune system). Copy number gains of PDL1 have been observed only in one case of metastatic BCC (113). The patient, who was otherwise resistant to vismodegib and sonidegib, demonstrated a dramatic response to nivolumab (an anti-PD-1 antibody blocking the PD-1/PD-L1 interaction), which strongly suggested that the copy number gain may be a biomarker of sensitivity to anti-PD-1/PD-L1 checkpoint treatments (113). It was also shown in an independent study that some patients (up to $\sim 40 \%$ ) with advanced BCC (not tested for PDL1 amplification) respond to pembrolizumab (another anti-PD-1 antibody) (113, 153). Therefore, assessment of copy number gains of the PDL1/ $P D L 2$ region may help to rationalize such treatment; however, further study with a larger group of samples is required.

Finally, we would like to note the apparent limitations of the study. As it was intended to be a preliminary evaluation of noncoding mutations in BCC, we analyzed only a small number of samples, and as such, we limited the characterization of the identified variants to computational analyses. It has to be also noted that our analysis covered only a small fraction $(\sim 1 \%)$ of the noncoding genome that cumulatively accounts for $\sim 98 \%$ of the genome and contains many different functional elements not covered in our analysis, including promoters, enhancers, and genes of different classes of non-coding RNAs.

In summary, in this study utilizing WES BCC data, we revealed not only mutations in coding regions of previously known BCC-related genes but also frequent mutations in noncoding regions of cancer-related genes, some of which may be strong candidates for new BCC drivers. Although the functional role of the individual identified genes/mutations requires further experimental interrogations, our results provide a strong basis for further analyses of noncoding variants in BCC and other cancer types.

\section{DATA AVAILABILITY STATEMENT}

The datasets presented in this study can be found in online repositories. The names of the repository/repositories and accession number(s) can be found below: https://www.ncbi. nlm.nih.gov/, PRJNA747148.

\section{ETHICS STATEMENT}

The studies involving human participants were reviewed and approved by bioethics committee of the Ruhr-University of Bochum. The patients/participants provided their written informed consent to participate in this study.

\section{AUTHOR CONTRIBUTIONS}

PN extracted the DNA samples, performed almost all experimental and computational analyses, drafted the manuscript (with $\mathrm{PK}$ ), prepared all figures, tables, and supplementary materials. PG-M participated in the study design, extracted mutations from TCGA, assisted PN in experimental analyses, participated in the manuscript preparation. MU-T prepared the scripts for mutation calling and mutations annotation, assisted $\mathrm{PN}$ in computational analyses, participated in the manuscript preparation. IM-T performed the analysis of the NFAT1 TF binding site in the promoter of DHODH, participated in the manuscript preparation. NS participated in CNA analysis with GISTIC2, participated in the manuscript preparation. AP assisted in computational analyses, participated in the manuscript preparation. LS - collected and characterized the BCC samples, participated in the manuscript preparation. MS - participated in conceiving the study, participated in collection and characterization of BCC samples, participated in the manuscript preparation. $\mathrm{PK}$ - conceived and supervised the study, drafted the manuscript (with PN), acquired the funding. All authors contributed to the article and approved the submitted version. 


\section{FUNDING}

This work was supported by research grants from the Polish National Science Centre [2016/22/A/NZ2/00184 and 2020/39/B/ NZ5/01970] (to PK).

\section{REFERENCES}

1. Samarasinghe V, Madan V, Lear JT. Focus on Basal Cell Carcinoma. J Skin Cancer (2011) 2011:328615. doi: 10.1155/2011/328615

2. Puig S, Berrocal A. Management of High-Risk and Advanced Basal Cell Carcinoma. Clin Transl Oncol Off Publ Fed Span Oncol Soc Natl Cancer Inst Mex (2015) 17:497-503. doi: 10.1007/s12094-014-1272-9

3. Pellegrini C, Maturo MG, Di Nardo L, Ciciarelli V, Gutiérrez García-Rodrigo C, Fargnoli MC. Understanding the Molecular Genetics of Basal Cell Carcinoma. Int J Mol Sci (2017) 18(11):2485. doi: 10.3390/ijms18112485

4. Evans DG, Howard E, Giblin C, Clancy T, Spencer H, Huson SM, et al. Birth Incidence and Prevalence of Tumor-Prone Syndromes: Estimates From a UK Family Genetic Register Service. Am J Med Genet A (2010) 152A:32732. doi: 10.1002/ajmg.a.33139

5. Sexton M, Jones DB, Maloney ME. Histologic Pattern Analysis of Basal Cell Carcinoma. Study of a Series of 1039 Consecutive Neoplasms. J Am Acad Dermatol (1990) 23:1118-26. doi: 10.1016/0190-9622(90)70344-h

6. Rippey JJ. Why Classify Basal Cell Carcinomas? Histopathology (1998) 32:393-8. doi: 10.1046/j.1365-2559.1998.00431.x

7. Raasch BA, Buettner PG, Garbe C. Basal Cell Carcinoma: Histological Classification and Body-Site Distribution. Br J Dermatol (2006) 155:4017. doi: 10.1111/j.1365-2133.2006.07234.x

8. Lo JS, Snow SN, Reizner GT, Mohs FE, Larson PO, Hruza GJ. Metastatic Basal Cell Carcinoma: Report of Twelve Cases With a Review of the Literature. J Am Acad Dermatol (1991) 24:715-9. doi: 10.1016/0190-9622 (91)70108-e

9. Ting PT, Kasper R, Arlette JP. Metastatic Basal Cell Carcinoma: Report of Two Cases and Literature Review. J Cutan Med Surg (2005) 9:10-5. doi: 10.1007/s10227-005-0027-1

10. Crowson AN, Magro CM, Kadin ME, Stranc M. Differential Expression of the Bcl-2 Oncogene in Human Basal Cell Carcinoma. Hum Pathol (1996) 27:355-9. doi: 10.1016/s0046-8177(96)90108-2

11. Crowson AN. Basal Cell Carcinoma: Biology, Morphology and Clinical Implications. Mod Pathol Off J U S Can Acad Pathol Inc (2006) 19 (Suppl 2): S127-147. doi: 10.1038/modpathol.3800512

12. Epstein EH. Basal Cell Carcinomas: Attack of the Hedgehog. Nat Rev Cancer (2008) 8:743-54. doi: 10.1038/nrc2503

13. Iwasaki JK, Srivastava D, Moy RL, Lin HJ, Kouba DJ. The Molecular Genetics Underlying Basal Cell Carcinoma Pathogenesis and Links to Targeted Therapeutics. J Am Acad Dermatol (2012) 66:e167-178. doi: 10.1016/j.jaad.2010.06.054

14. Jayaraman SS, Rayhan DJ, Hazany S, Kolodney MS. Mutational Landscape of Basal Cell Carcinomas by Whole-Exome Sequencing. J Invest Dermatol (2014) 134:213-20. doi: 10.1038/jid.2013.276

15. Bonilla X, Parmentier L, King B, Bezrukov F, Kaya G, Zoete V, et al. Genomic Analysis Identifies New Drivers and Progression Pathways in Skin Basal Cell Carcinoma. Nat Genet (2016) 48:398-406. doi: 10.1038/ng.3525

16. Verkouteren JAC, Ramdas KHR, Wakkee M, Nijsten T. Epidemiology of Basal Cell Carcinoma: Scholarly Review. Br J Dermatol (2017) 177:359-72. doi: 10.1111/bjd.15321

17. Peris K, Fargnoli MC, Garbe C, Kaufmann R, Bastholt L, Seguin NB, et al. Diagnosis and Treatment of Basal Cell Carcinoma: European ConsensusBased Interdisciplinary Guidelines. Eur J Cancer Oxf Engl (1990) 2019:118: 10-34. doi: 10.1016/j.ejca.2019.06.003

18. Gailani MR, Leffell DJ, Ziegler A, Gross EG, Brash DE, Bale AE. Relationship Between Sunlight Exposure and a Key Genetic Alteration in Basal Cell Carcinoma. J Natl Cancer Inst (1996) 88:349-54. doi: 10.1093/ jnci/88.6.349

19. Maloney ME. Arsenic in Dermatology. Dermatol Surg Off Publ Am Soc Dermatol Surg Al (1996) 22:301-4. doi: 10.1111/j.1524-4725.1996.tb00322.x

\section{SUPPLEMENTARY MATERIAL}

The Supplementary Material for this article can be found online at: https://www.frontiersin.org/articles/10.3389/fonc.2021. 752579/full\#supplementary-material

20. Rubin AI, Chen EH, Ratner D. Basal-Cell Carcinoma. N Engl J Med (2005) 353:2262-9. doi: 10.1056/NEJMra044151

21. Palacios-Álvarez I, González-Sarmiento R, Fernández-López E. Gorlin Syndrome. Actas Dermo Sifiliográficas Engl Ed (2018) 109:207-17. doi: 10.1016/j.adengl.2018.02.002

22. Diederichs S, Bartsch L, Berkmann JC, Fröse K, Heitmann J, Hoppe C, et al. The Dark Matter of the Cancer Genome: Aberrations in Regulatory Elements, Untranslated Regions, Splice Sites, non-Coding RNA and Synonymous Mutations. EMBO Mol Med (2016) 8:442-57. doi: 10.15252/emmm.201506055

23. Leppek K, Das R, Barna M. Functional 5' UTR mRNA Structures in Eukaryotic Translation Regulation and How to Find Them. Nat Rev Mol Cell Biol (2018) 19:158-74. doi: 10.1038/nrm.2017.103

24. Lord J, Gallone G, Short PJ, McRae JF, Ironfield H, Wynn EH, et al Pathogenicity and Selective Constraint on Variation Near Splice Sites. Genome Res (2019) 29:159-70. doi: 10.1101/gr.238444.118

25. Horn S, Figl A, Rachakonda PS, Fischer C, Sucker A, Gast A, et al. TERT Promoter Mutations in Familial and Sporadic Melanoma. Science (2013) 339:959-61. doi: 10.1126/science.1230062

26. Huang FW, Hodis E, Xu MJ, Kryukov GV, Chin L, Garraway LA. Highly Recurrent TERT Promoter Mutations in Human Melanoma. Science (2013) 339:957-9. doi: 10.1126/science.1229259

27. Maturo MG, Rachakonda S, Heidenreich B, Pellegrini C, Srinivas N Requena C, et al. Coding and Noncoding Somatic Mutations in Candidate Genes in Basal Cell Carcinoma. Sci Rep (2020) 10:8005. doi: 10.1038/s41598020-65057-2

28. Urbanek-Trzeciak MO, Galka-Marciniak P, Nawrocka PM, Kowal E, Szwec S, Giefing M, et al. Pan-Cancer Analysis of Somatic Mutations in miRNA Genes. EBioMedicine (2020) 61:103051. doi: 10.1016/j.ebiom.2020.103051

29. Sand M, Skrygan M, Sand D, Georgas D, Hahn SA, Gambichler T, et al Expression of microRNAs in Basal Cell Carcinoma. Br J Dermatol (2012) 167:847-55. doi: 10.1111/j.1365-2133.2012.11022.x

30. Sand M, Hessam S, Amur S, Skrygan M, Bromba M, Stockfleth E, et al. Expression of Oncogenic miR-17-92 and Tumor Suppressive miR-143-145 Clusters in Basal Cell Carcinoma and Cutaneous Squamous Cell Carcinoma. J Dermatol Sci (2017) 86:142-8. doi: 10.1016/j.jdermsci.2017.01.012

31. Sand M, Bromba A, Sand D, Gambichler T, Hessam S, Becker JC, et al Dicer Sequencing, Whole Genome Methylation Profiling, mRNA and smallRNA Sequencing Analysis in Basal Cell Carcinoma. Cell Physiol Biochem Int J Exp Cell Physiol Biochem Pharmacol (2019) 53:760-73. doi: $10.33594 / 000000171$

32. Rheinbay E, Nielsen MM, Abascal F, Wala JA, Shapira O, Tiao G, et al. Analyses of non-Coding Somatic Drivers in 2,658 Cancer Whole Genomes. Nature (2020) 578:102-11. doi: 10.1038/s41586-020-1965-x

33. Sakthikumar S, Roy A, Haseeb L, Pettersson ME, Sundström E, Marinescu VD, et al. Whole-Genome Sequencing of Glioblastoma Reveals Enrichment of non-Coding Constraint Mutations in Known and Novel Genes. Genome Biol (2020) 21:127. doi: 10.1186/s13059-020-02035-x

34. Jiang H, Lei R, Ding S-W, Zhu S. Skewer: A Fast and Accurate Adapter Trimmer for Next-Generation Sequencing Paired-End Reads. BMC Bioinf (2014) 15:182. doi: 10.1186/1471-2105-15-182

35. Díaz-Gay M, Vila-Casadesús M, Franch-Expósito S, Hernández-Illán E, Lozano JJ, Castellví-Bel S. Mutational Signatures in Cancer (MuSiCa): A Web Application to Implement Mutational Signatures Analysis in Cancer Samples. BMC Bioinf (2018) 19:224. doi: 10.1186/s12859-018-2234-y

36. Alexandrov LB, Nik-Zainal S, Wedge DC, Aparicio SAJR, Behjati S, Biankin $\mathrm{AV}$, et al. Signatures of Mutational Processes in Human Cancer. Nature (2013) 500:415-21. doi: 10.1038/nature 12477

37. Lawrence MS, Stojanov P, Polak P, Kryukov GV, Cibulskis K, Sivachenko A, et al. Mutational Heterogeneity in Cancer and the Search for New CancerAssociated Genes. Nature (2013) 499:214-8. doi: 10.1038/nature12213 
38. Cingolani P, Platts A, Wang LL, Coon M, Nguyen T, Wang L, et al. A Program for Annotating and Predicting the Effects of Single Nucleotide Polymorphisms, SnpEff. Fly (Austin) (2012) 6:80-92. doi: 10.4161/ fly.19695

39. Mularoni L, Sabarinathan R, Deu-Pons J, Gonzalez-Perez A, López-Bigas N. OncodriveFML: A General Framework to Identify Coding and non-Coding Regions With Cancer Driver Mutations. Genome Biol (2016) 17:128. doi: 10.1186/s13059-016-0994-0

40. Mermel CH, Schumacher SE, Hill B, Meyerson ML, Beroukhim R, Getz G. GISTIC2.0 Facilitates Sensitive and Confident Localization of the Targets of Focal Somatic Copy-Number Alteration in Human Cancers. Genome Biol (2011) 12:R41. doi: 10.1186/gb-2011-12-4-r41

41. Kozlowski P, Roberts P, Dabora S, Franz D, Bissler J, Northrup H, et al. Identification of 54 Large Deletions/Duplications in TSC1 and TSC2 Using MLPA, and Genotype-Phenotype Correlations. Hum Genet (2007) 121:389400. doi: 10.1007/s00439-006-0308-9

42. Marcinkowska M, Wong K-K, Kwiatkowski DJ, Kozlowski P. Design and Generation of MLPA Probe Sets for Combined Copy Number and SmallMutation Analysis of Human Genes: EGFR as an Example. Sci World J (2010) 10:2003-18. doi: 10.1100/tsw.2010.195

43. Galka-Marciniak P, Urbanek-Trzeciak MO, Nawrocka PM, Kozlowski P. A Pan-Cancer Atlas of Somatic Mutations in miRNA Biogenesis Genes. Nucleic Acids Res (2021) 49:601-20. doi: 10.1093/nar/gkaa1223

44. Zhou X, Edmonson MN, Wilkinson MR, Patel A, Wu G, Liu Y, et al. Exploring Genomic Alteration in Pediatric Cancer Using ProteinPaint. Nat Genet (2016) 48:4-6. doi: 10.1038/ng.3466

45. UniProt Consortium. UniProt: A Worldwide Hub of Protein Knowledge. Nucleic Acids Res (2019) 47:D506-15. doi: 10.1093/nar/gky1049

46. Crowdis J, He MX, Reardon B, Van Allen EM. CoMut: Visualizing Integrated Molecular Information With Comutation Plots. Bioinformatics (2020) 36:4348-9. doi: 10.1093/bioinformatics/btaa554

47. Lewis BP, Burge CB, Bartel DP. Conserved Seed Pairing, Often Flanked by Adenosines, Indicates That Thousands of Human Genes are microRNA Targets. Cell (2005) 120:15-20. doi: 10.1016/j.cell.2004.12.035

48. Zuker M. Mfold Web Server for Nucleic Acid Folding and Hybridization Prediction. Nucleic Acids Res (2003) 31:3406-15. doi: 10.1093/nar/gkg595

49. Huang H-Y, Chien C-H, Jen K-H, Huang H-D. RegRNA: An Integrated Web Server for Identifying Regulatory RNA Motifs and Elements. Nucleic Acids Res (2006) 34:W429-434. doi: 10.1093/nar/gkl333

50. Daily K, Patel VR, Rigor P, Xie X, Baldi P. MotifMap: Integrative GenomeWide Maps of Regulatory Motif Sites for Model Species. BMC Bioinf (2011) 12:495. doi: 10.1186/1471-2105-12-495

51. Supek F, Miñana B, Valcárcel J, Gabaldón T, Lehner B. Synonymous Mutations Frequently Act as Driver Mutations in Human Cancers. Cell (2014) 156:1324-35. doi: 10.1016/j.cell.2014.01.051

52. Lawrence MS, Stojanov P, Mermel CH, Garraway LA, Golub TR, Meyerson $\mathrm{M}$, et al. Discovery and Saturation Analysis of Cancer Genes Across 21 Tumor Types. Nature (2014) 505:495-501. doi: 10.1038/nature12912

53. Alexandrov LB, Kim J, Haradhvala NJ, Huang MN, Tian Ng AW, Wu Y, et al. The Repertoire of Mutational Signatures in Human Cancer. Nature (2020) 578:94-101. doi: 10.1038/s41586-020-1943-3

54. Bin Y, Wang X, Zhao L, Wen P, Xia J. An Analysis of Mutational Signatures of Synonymous Mutations Across 15 Cancer Types. BMC Med Genet (2019) 20:190. doi: 10.1186/s12881-019-0926-4

55. Sharma Y, Miladi M, Dukare S, Boulay K, Caudron-Herger M, Groß M, et al. A Pan-Cancer Analysis of Synonymous Mutations. Nat Commun (2019) 10:2569. doi: 10.1038/s41467-019-10489-2

56. Hase K, Kimura S, Takatsu H, Ohmae M, Kawano S, Kitamura H, et al. MSec Promotes Membrane Nanotube Formation by Interacting With Ral and the Exocyst Complex. Nat Cell Biol (2009) 11:1427-32. doi: 10.1038/ncb1990

57. Jia L, Zhou Z, Liang H, Wu J, Shi P, Li F, et al. KLF5 Promotes Breast Cancer Proliferation, Migration and Invasion in Part by Upregulating the Transcription of TNFAIP2. Oncogene (2016) 35:2040-51. doi: 10.1038/ onc.2015.263

58. Xie Y, Wang B. Downregulation of TNFAIP2 Suppresses Proliferation and Metastasis in Esophageal Squamous Cell Carcinoma Through Activation of the Wnt/ $\beta$-Catenin Signaling Pathway. Oncol Rep (2017) 37:2920-8. doi: 10.3892/or.2017.5557
59. Jia L, Shi Y, Wen Y, Li W, Feng J, Chen C. The Roles of TNFAIP2 in Cancers and Infectious Diseases. J Cell Mol Med (2018) 22:5188-95. doi: 10.1111/ jcmm.13822

60. Kodach LL, Jacobs RJ, Heijmans J, van Noesel CJM, Langers AMJ, Verspaget HW, et al. The Role of EZH2 and DNA Methylation in the Silencing of the Tumour Suppressor RUNX3 in Colorectal Cancer. Carcinogenesis (2010) 31:1567-75. doi: 10.1093/carcin/bgq147

61. Xie Q, Wang H, Heilman ER, Walsh MG, Haseeb MA, Gupta R. Increased Expression of Enhancer of Zeste Homolog 2 (EZH2) Differentiates Squamous Cell Carcinoma From Normal Skin and Actinic Keratosis. Eur J Dermatol EJD (2014) 24:41-5. doi: 10.1684/ejd.2013.2219

62. Rao RC, Chan MP, Andrews CA, Kahana A. EZH2, Proliferation Rate, and Aggressive Tumor Subtypes in Cutaneous Basal Cell Carcinoma. JAMA Oncol (2016) 2:962-3. doi: 10.1001/jamaoncol.2016.0021

63. Bödör C, Grossmann V, Popov N, Okosun J, O'Riain C, Tan K, et al. EZH2 Mutations are Frequent and Represent an Early Event in Follicular Lymphoma. Blood (2013) 122:3165-8. doi: 10.1182/blood-201304-496893

64. Donaldson-Collier MC, Sungalee S, Zufferey M, Tavernari D, Katanayeva N, Battistello E, et al. EZH2 Oncogenic Mutations Drive Epigenetic, Transcriptional, and Structural Changes Within Chromatin Domains. Nat Genet (2019) 51:517-28. doi: 10.1038/s41588-018-0338-y

65. Weaver TM, Liu J, Connelly KE, Coble C, Varzavand K, Dykhuizen EC, et al. The EZH2 SANT1 Domain is a Histone Reader Providing Sensitivity to the Modification State of the H4 Tail. Sci Rep (2019) 9:987. doi: 10.1038/s41598018-37699-w

66. Fang L, Seki A, Fang G. SKAP Associates With Kinetochores and Promotes the Metaphase-to-Anaphase Transition. Cell Cycle (2009) 8:2819-27. doi: $10.4161 /$ cc.8.17.9514

67. Lee CS, Bhaduri A, Mah A, Johnson WL, Ungewickell A, Aros CJ, et al. Recurrent Point Mutations in the Kinetochore Gene KNSTRN in Cutaneous Squamous Cell Carcinoma. Nat Genet (2014) 46:1060-2. doi: 10.1038/ ng.3091

68. Jaju PD, Nguyen CB, Mah AM, Atwood SX, Li J, Zia A, et al. Mutations in the Kinetochore Gene KNSTRN in Basal Cell Carcinoma. J Invest Dermatol (2015) 135:3197-200. doi: 10.1038/jid.2015.339

69. Lu S, Wang R, Cai C, Liang J, Xu L, Miao S, et al. Small Kinetochore Associated Protein (SKAP) Promotes UV-Induced Cell Apoptosis Through Negatively Regulating pre-mRNA Processing Factor 19 (Prp19). PloS One (2014) 9:e92712. doi: 10.1371/journal.pone.0092712

70. Marchion DC, Cottrill HM, Xiong Y, Chen N, Bicaku E, Fulp WJ, et al. BAD Phosphorylation Determines Ovarian Cancer Chemosensitivity and Patient Survival. Clin Cancer Res (2011) 17:6356-66. doi: 10.1158/1078-0432.CCR11-0735

71. Stickles XB, Marchion DC, Bicaku E, Al Sawah E, Abbasi F, Xiong Y, et al. BADMediated Apoptotic Pathway is Associated With Human Cancer Development. Int J Mol Med (2015) 35:1081-7. doi: 10.3892/ijmm.2015.2091

72. Hosseini M, Dousset L, Mahfouf W, Serrano-Sanchez M, Redonnet-Vernhet I, Mesli S, et al. Energy Metabolism Rewiring Precedes UVB-Induced Primary Skin Tumor Formation. Cell Rep (2018) 23:3621-34. doi: 10.1016/j.celrep.2018.05.060

73. Hosseini M, Dousset L, Michon P, Mahfouf W, Muzotte E, Bergeron V, et al. UVB-Induced DHODH Upregulation, Which is Driven by STAT3, is a Promising Target for Chemoprevention and Combination Therapy of Photocarcinogenesis. Oncogenesis (2019) 8:52. doi: 10.1038/s41389-0190161-z

74. Hayward NK, Wilmott JS, Waddell N, Johansson PA, Field MA, Nones K, et al. Whole-Genome Landscapes of Major Melanoma Subtypes. Nature (2017) 545:175-80. doi: 10.1038/nature22071

75. Dutton-Regester K, Gartner JJ, Emmanuel R, Qutob N, Davies MA, Gershenwald JE, et al. A Highly Recurrent RPS27 5'UTR Mutation in Melanoma. Oncotarget (2014) 5:2912-7. doi: 10.18632/oncotarget.2048

76. Floristán A, Morales L, Hanniford D, Martinez C, Castellano-Sanz E, Dolgalev I, et al. Functional Analysis of RPS27 Mutations and Expression in Melanoma. Pigment Cell Melanoma Res (2020) 33:466-79. doi: 10.1111/ pcmr.12841

77. Huang H-Y, Lin Y-C-D, Li J, Huang K-Y, Shrestha S, Hong H-C, et al. Mirtarbase 2020: Updates to the Experimentally Validated microRNA- 
Target Interaction Database. Nucleic Acids Res (2020) 48:D148-54. doi: $10.1093 /$ nar/gkz896

78. Salamov AA, Nishikawa T, Swindells MB. Assessing Protein Coding Region Integrity in cDNA Sequencing Projects. Bioinforma Oxf Engl (1998) 14:38490. doi: 10.1093/bioinformatics/14.5.384

79. Peitsch MC. Large Scale Protein Modelling and Model Repository. Proc Int Conf Intell Syst Mol Biol (1997) 5:234-6.

80. Chang T-H, Huang H-Y, Hsu JB-K, Weng S-L, Horng J-T, Huang H-D. An Enhanced Computational Platform for Investigating the Roles of Regulatory RNA and for Identifying Functional RNA Motifs. BMC Bioinf (2013) 14:S4. doi: 10.1186/1471-2105-14-S2-S4

81. Shoshan E, Braeuer RR, Kamiya T, Mobley AK, Huang L, Vasquez ME, et al. NFAT1 Directly Regulates IL8 and MMP3 to Promote Melanoma Tumor Growth and Metastasis. Cancer Res (2016) 76:3145-55. doi: 10.1158/00085472.CAN-15-2511

82. Shen Y, Song Z, Lu X, Ma Z, Lu C, Zhang B, et al. Fas Signaling-Mediated T H 9 Cell Differentiation Favors Bowel Inflammation and Antitumor Functions. Nat Commun (2019) 10:2924. doi: 10.1038/s41467-019-10889-4

83. Wang NJ, Sanborn Z, Arnett KL, Bayston LJ, Liao W, Proby CM, et al. LossOf-Function Mutations in Notch Receptors in Cutaneous and Lung Squamous Cell Carcinoma. Proc Natl Acad Sci (2011) 108:17761-6. doi: 10.1073/pnas.1114669108

84. Purdie KJ, Lambert SR, Teh M-T, Chaplin T, Molloy G, Raghavan M, et al. Allelic Imbalances and Microdeletions Affecting the PTPRD Gene in Cutaneous Squamous Cell Carcinomas Detected Using Single Nucleotide Polymorphism Microarray Analysis. Genes Chromosomes Cancer (2007) 46:661-9. doi: 10.1002/gcc.20447

85. Veeriah S, Brennan C, Meng S, Singh B, Fagin JA, Solit DB, et al. The Tyrosine Phosphatase PTPRD is a Tumor Suppressor That is Frequently Inactivated and Mutated in Glioblastoma and Other Human Cancers. Proc Natl Acad Sci (2009) 106:9435-40. doi: 10.1073/pnas.0900571106

86. Walia V, Prickett TD, Kim J-S, Gartner JJ, Lin JC, Zhou M, et al. Mutational and Functional Analysis of the Tumor-Suppressor PTPRD in Human Melanoma. Hum Mutat (2014) 35:1301-10. doi: 10.1002/humu.22630

87. Peyser ND, Du Y, Li H, Lui V, Xiao X, Chan TA, et al. Loss-Of-Function PTPRD Mutations Lead to Increased STAT3 Activation and Sensitivity to STAT3 Inhibition in Head and Neck Cancer. PloS One (2015) 10:e0135750. doi: 10.1371/journal.pone.0135750

88. Szaumkessel M, Wojciechowska S, Janiszewska J, Zemke N, Byzia E, Kiwerska K, et al. Recurrent Epigenetic Silencing of the PTPRD Tumor Suppressor in Laryngeal Squamous Cell Carcinoma. Tumor Biol (2017) 39:1010428317691427. doi: 10.1177/1010428317691427

89. Adib E, Klonowska K, Giannikou K, Do KT, Pruitt-Thompson S, Bhushan $\mathrm{K}$, et al. Phase II Clinical Trial of Everolimus in a Pan-Cancer Cohort of Patients With mTOR Pathway Alterations. Clin Cancer Res (2021) 27 (14):3845-53. doi: 10.1158/1078-0432.CCR-20-4548

90. Wu Z, Hansmann B, Meyer-Hoffert U, Gläser R, Schröder J-M. Molecular Identification and Expression Analysis of Filaggrin-2, a Member of the S100 Fused-Type Protein Family. PloS One (2009) 4(4):e5227. doi: 10.1371/ journal.pone.0005227

91. Leman G, Moosbrugger-Martinz V, Blunder S, Pavel P, Dubrac S. 3dOrganotypic Cultures to Unravel Molecular and Cellular Abnormalities in Atopic Dermatitis and Ichthyosis Vulgaris. Cells (2019) 8(5):489. doi: $10.3390 /$ cells 8050489

92. Liu H, Toman RE, Goparaju SK, Maceyka M, Nava VE, Sankala H, et al. Sphingosine Kinase Type 2 is a Putative BH3-Only Protein That Induces Apoptosis. J Biol Chem (2003) 278:40330-6. doi: 10.1074/ jbc.M304455200

93. Igarashi N, Okada T, Hayashi S, Fujita T, Jahangeer S, Nakamura S. Sphingosine Kinase 2 is a Nuclear Protein and Inhibits DNA Synthesis. J Biol Chem (2003) 278:46832-9. doi: 10.1074/jbc.M306577200

94. Maceyka M, Sankala H, Hait NC, Le Stunff H, Liu H, Toman R, et al. SphK1 and SphK2, Sphingosine Kinase Isoenzymes With Opposing Functions in Sphingolipid Metabolism. J Biol Chem (2005) 280:37118-29. doi: 10.1074/ jbc.M502207200

95. Van Brocklyn JR, Jackson CA, Pearl DK, Kotur MS, Snyder PJ, Prior TW. Sphingosine Kinase-1 Expression Correlates With Poor Survival of Patients With Glioblastoma Multiforme: Roles of Sphingosine Kinase Isoforms in
Growth of Glioblastoma Cell Lines. J Neuropathol Exp Neurol (2005) 64:695-705. doi: 10.1097/01.jnen.0000175329.59092.2c

96. Gao P, Smith CD. Ablation of Sphingosine Kinase-2 Inhibits Tumor Cell Proliferation and Migration. Mol Cancer Res MCR (2011) 9:1509-19. doi: 10.1158/1541-7786.MCR-11-0336

97. Su X, Malouf GG, Chen Y, Zhang J, Yao H, Valero V, et al. Comprehensive Analysis of Long non-Coding RNAs in Human Breast Cancer Clinical Subtypes. Oncotarget (2014) 5:9864-76. doi: 10.18632/oncotarget.2454

98. Segers VFM, Dugaucquier L, Feyen E, Shakeri H, De Keulenaer GW. The Role of ErbB4 in Cancer. Cell Oncol Dordr (2020) 43:335-52. doi: 10.1007/ s13402-020-00499-4

99. Sand M, Gambichler T, Skrygan M, Sand D, Scola N, Altmeyer P, et al. Expression Levels of the microRNA Processing Enzymes Drosha and Dicer in Epithelial Skin Cancer. Cancer Invest (2010) 28:649-53. doi: 10.3109/ 07357901003630918

100. Griewank KG, Murali R, Schilling B, Schimming T, Möller I, Moll I, et al. TERT Promoter Mutations are Frequent in Cutaneous Basal Cell Carcinoma and Squamous Cell Carcinoma. PloS One (2013) 8:e80354. doi: 10.1371/ journal.pone.0080354

101. Scott GA, Laughlin TS, Rothberg PG. Mutations of the TERT Promoter are Common in Basal Cell Carcinoma and Squamous Cell Carcinoma. Mod Pathol Off J U S Can Acad Pathol Inc (2014) 27:516-23. doi: 10.1038/ modpathol.2013.167

102. Di Nardo L, Pellegrini C, Di Stefani A, Ricci F, Fossati B, Del Regno L, et al. Molecular Alterations in Basal Cell Carcinoma Subtypes. Sci Rep (2021) 11:13206. doi: 10.1038/s41598-021-92592-3

103. Malhotra N, Leyva-Castillo JM, Jadhav U, Barreiro O, Kam C, O’Neill NK, et al. Ror $\alpha$-Expressing T Regulatory Cells Restrain Allergic Skin Inflammation. Sci Immunol (2018) 3(21):eaa06923. doi: 10.1126/ sciimmunol.aao6923

104. Houle AA, Gibling H, Lamaze FC, Edgington HA, Soave D, Fave M-J, et al. Aberrant PRDM9 Expression Impacts the Pan-Cancer Genomic Landscape. Genome Res (2018) 28:1611-20. doi: 10.1101/gr.231696.117

105. Nicolai S, Mahen R, Raschellà G, Marini A, Pieraccioli M, Malewicz M, et al. ZNF281 is Recruited on DNA Breaks to Facilitate DNA Repair by nonHomologous End Joining. Oncogene (2020) 39:754-66. doi: 10.1038/s41388019-1028-7

106. Pasparakis M, Courtois G, Hafner M, Schmidt-Supprian M, Nenci A, Toksoy A, et al. TNF-Mediated Inflammatory Skin Disease in Mice With EpidermisSpecific Deletion of IKK2. Nature (2002) 417:861-6. doi: 10.1038/ nature 00820

107. Stratis A, Pasparakis M, Markur D, Knaup R, Pofahl R, Metzger D, et al. Localized Inflammatory Skin Disease Following Inducible Ablation of I Kappa B Kinase 2 in Murine Epidermis. J Invest Dermatol (2006) 126:61420. doi: $10.1038 /$ sj.jid. 5700092

108. Cornish GH, Tung SL, Marshall D, Ley S, Seddon BP. Tissue Specific Deletion of Inhibitor of Kappa B Kinase 2 With OX40-Cre Reveals the Unanticipated Expression From the OX40 Locus in Skin Epidermis. PloS One (2012) 7(2):e32193. doi: 10.1371/journal.pone.0032193

109. Kirkley KS, Walton KD, Duncan C, Tjalkens RB. Spontaneous Development of Cutaneous Squamous Cell Carcinoma in Mice With Cell-Specific Deletion of Inhibitor of кb Kinase 2. Comp Med (2017) 67:407-15.

110. Boyle GM, Woods SL, Bonazzi VF, Stark MS, Hacker E, Aoude LG, et al. Melanoma Cell Invasiveness is Regulated by miR-211 Suppression of the BRN2 Transcription Factor. Pigment Cell Melanoma Res (2011) 24:525-37. doi: 10.1111/j.1755-148X.2011.00849.x

111. Simmons JL, Pierce CJ, Al-Ejeh F, Boyle GM. MITF and BRN2 Contribute to Metastatic Growth After Dissemination of Melanoma. Sci Rep (2017) 7:10909. doi: 10.1038/s41598-017-11366-y

112. Zhao G, Wei Z, Guo Y. MicroRNA-107 is a Novel Tumor Suppressor Targeting POU3F2 in Melanoma. Biol Res (2020) 53:11. doi: 10.1186/s40659020-00278-3

113. Ikeda S, Goodman AM, Cohen PR, Jensen TJ, Ellison CK, Frampton G, et al. Metastatic Basal Cell Carcinoma With Amplification of PD-L1: Exceptional Response to Anti-PD1 Therapy. NPJ Genomic Med (2016) 1:1-5. doi: 10.1038/npjgenmed.2016.37

114. Chandramouli A, Shi J, Feng Y, Holubec H, M.Shanas R, Bhattacharyya AK, et al. Haploinsufficiency of the Cdc2l Gene Contributes to Skin Cancer 
Development in Mice. Carcinogenesis (2007) 28:2028-35. doi: 10.1093/ carcin/bgm066

115. de Cid R, Riveira-Munoz E, Zeeuwen PLJM, Robarge J, Liao W, Dannhauser EN, et al. Deletion of the Late Cornified Envelope LCE3B and LCE3C Genes as a Susceptibility Factor for Psoriasis. Nat Genet (2009) 41:211-5. doi: $10.1038 /$ ng.313

116. Myskowski PL, Pollack MS, Schorr E, Dupont B, Safai B. Human Leukocyte Antigen Associations in Basal Cell Carcinoma. J Am Acad Dermatol (1985) 12:997-1000. doi: 10.1016/s0190-9622(85)70127-2

117. Hua LA, Kagen CN, Carpenter RJ, Goltz RW. HLA and Beta 2Microglobulin Expression in Basal and Squamous Cell Carcinomas of the Skin. Int J Dermatol (1985) 24:660-3. doi: 10.1111/j.13654362.1985.tb05719.x

118. Markey AC, Churchill LJ, MacDonald DM. Altered Expression of Major Histocompatibility Complex (MHC) Antigens by Epidermal Tumours. J Cutan Pathol (1990) 17:65-71. doi: 10.1111/j.1600-0560.1990.tb00058.x

119. Streilein JW. Immunogenetic Factors in Skin Cancer. N Engl J Med (1991) 325:884-7. doi: 10.1056/NEJM199109193251210

120. García-Plata D, Mozos E, Carrasco L, Solana R. HLA Molecule Expression in Cutaneous Squamous Cell Carcinomas: An Immunopathological Study and Clinical-Immunohistopathological Correlations. Histol Histopathol (1993) 8:219-26.

121. Johnson DB, Estrada MV, Salgado R, Sanchez V, Doxie DB, Opalenik SR, et al. Melanoma-Specific MHC-II Expression Represents a TumourAutonomous Phenotype and Predicts Response to Anti-PD-1/PD-L1 Therapy. Nat Commun (2016) 7:10582. doi: 10.1038/ncomms10582

122. Chen Y-Y, Chang W-A, Lin E-S, Chen Y-J, Kuo P-L. Expressions of HLA Class II Genes in Cutaneous Melanoma Were Associated With Clinical Outcome: Bioinformatics Approaches and Systematic Analysis of Public Microarray and RNA-Seq Datasets. Diagnostics (2019) 9(2):59. doi: 10.3390/ diagnostics 9020059

123. Stanisz H, Saul S, Müller CSL, Kappl R, Niemeyer BA, Vogt T, et al. Inverse Regulation of Melanoma Growth and Migration by Orai1/STIM2Dependent Calcium Entry. Pigment Cell Melanoma Res (2014) 27:442-53. doi: $10.1111 / \mathrm{pcmr} .12222$

124. Isvoranu G, Surcel M, Huică R-I, Munteanu AN, Pîrvu IR, Ciotaru D, et al. Natural Killer Cell Monitoring in Cutaneous Melanoma - New Dynamic Biomarker. Oncol Lett (2019) 17:4197-206. doi: 10.3892/ol.2019.10069

125. Sanchez-Canteli M, Hermida-Prado F, Sordo-Bahamonde C, MontoroJiménez I, Pozo-Agundo E, Allonca E, et al. Lectin-Like Transcript 1 (LLT1) Checkpoint: A Novel Independent Prognostic Factor in HPVNegative Oropharyngeal Squamous Cell Carcinoma. Biomedicines (2020) 8 (12):535. doi: 10.3390/biomedicines 8120535

126. Lone MA, Hülsmeier AJ, Saied EM, Karsai G, Arenz C, von Eckardstein A, et al. Subunit Composition of the Mammalian Serine-Palmitoyltransferase Defines the Spectrum of Straight and Methyl-Branched Long-Chain Bases. Proc Natl Acad Sci (2020) 117:15591-8. doi: 10.1073/pnas.2002391117

127. Thomas NE, Edmiston SN, Tsai YS, Parker JS, Googe PB, Busam KJ, et al. Utility of TERT Promoter Mutations for Cutaneous Primary Melanoma Diagnosis. Am J Dermatopathol (2019) 41:264-72. doi: 10.1097/ DAD.0000000000001259

128. Shaughnessy M, Njauw C-N, Artomov M, Tsao H. Classifying Melanoma by TERT Promoter Mutational Status. J Invest Dermatol (2020) 140:390-394.e1. doi: 10.1016/j.jid.2019.06.149

129. Yang E, Zha J, Jockel J, Boise LH, Thompson CB, Korsmeyer SJ. Bad, a Heterodimeric Partner for Bcl-xL and Bcl-2, Displaces Bax and Promotes Cell Death. Cell (1995) 80:285-91. doi: 10.1016/0092-8674(95)90411-5

130. Datta SR, Dudek H, Tao X, Masters S, Fu H, Gotoh Y, et al. Akt Phosphorylation of BAD Couples Survival Signals to the Cell-Intrinsic Death Machinery. Cell (1997) 91:231-41. doi: 10.1016/s0092-8674(00) 80405-5

131. Sastry KSR, Al-Muftah MA, Li P, Al-Kowari MK, Wang E, Ismail Chouchane A, et al. Targeting Proapoptotic Protein BAD Inhibits Survival and Self-Renewal of Cancer Stem Cells. Cell Death Differ (2014) 21:1936-49. doi: 10.1038/cdd.2014.140

132. Sastry KS, Ibrahim WN, Chouchane AI. Multiple Signaling Pathways Converge on Proapoptotic Protein BAD to Promote Survival of Melanocytes. FASEB J (2020) 34:14602-14. doi: 10.1096/fj.202001260RR
133. Vander Heiden MG, Li XX, Gottleib E, Hill RB, Thompson CB, Colombini M. Bcl-xL Promotes the Open Configuration of the VoltageDependent Anion Channel and Metabolite Passage Through the Outer Mitochondrial Membrane. J Biol Chem (2001) 276:19414-9. doi: 10.1074/ jbc.M101590200

134. Danial NN, Gramm CF, Scorrano L, Zhang C-Y, Krauss S, Ranger AM, et al. BAD and Glucokinase Reside in a Mitochondrial Complex That Integrates Glycolysis and Apoptosis. Nature (2003) 424:952-6. doi: 10.1038/ nature 01825

135. Seo SY, Chen Y, Ivanovska I, Ranger AM, Hong SJ, Dawson VL, et al. BAD Is a Pro-Survival Factor Prior to Activation of Its Pro-Apoptotic Function*. J Biol Chem (2004) 279:42240-9. doi: 10.1074/jbc.M406775200

136. Danial NN, Walensky LD, Zhang C-Y, Choi CS, Fisher JK, Molina AJA, et al. Dual Role of Proapoptotic BAD in Insulin Secretion and Beta Cell Survival. Nat Med (2008) 14:144-53. doi: 10.1038/nm1717

137. Roy SS, Madesh M, Davies E, Antonsson B, Danial N, Hajnóczky G. Bad Targets the Permeability Transition Pore Independent of Bax or Bak to Switch Between Ca2+-Dependent Cell Survival and Death. Mol Cell (2009) 33:377-88. doi: 10.1016/j.molcel.2009.01.018

138. Berman SB, Chen Y, Qi B, McCaffery JM, Rucker EB, Goebbels S, et al. Bcl-X L Increases Mitochondrial Fission, Fusion, and Biomass in Neurons. J Cell Biol (2009) 184:707-19. doi: 10.1083/jcb.200809060

139. Aouacheria A, Baghdiguian S, Lamb HM, Huska JD, Pineda FJ, Hardwick JM. Connecting Mitochondrial Dynamics and Life-or-Death Events via Bcl2 Family Proteins. Neurochem Int (2017) 109:141-61. doi: 10.1016/j.neuint. 2017.04.009

140. Tomková H, Fujimoto W, Arata J. Expression of the Bcl-2 Homologue Bax in Normal Human Skin, Psoriasis Vulgaris and non-Melanoma Skin Cancers. Eur J Dermatol EJD (1998) 8:256-60.

141. Karst AM, Dai DL, Martinka M, Li G. PUMA Expression is Significantly Reduced in Human Cutaneous Melanomas. Oncogene (2005) 24:1111-6. doi: 10.1038/sj.onc.1208374

142. Aras S, Bai M, Lee I, Springett R, Hüttemann M, Grossman LI. MNRR1 (Formerly CHCHD2) is a Bi-Organellar Regulator of Mitochondrial Metabolism. Mitochondrion (2015) 20:43-51. doi: 10.1016/ j.mito.2014.10.003

143. Liu Y, Clegg HV, Leslie PL, Di J, Tollini LA, He Y, et al. CHCHD2 Inhibits Apoptosis by Interacting With Bcl-X L to Regulate Bax Activation. Cell Death Differ (2015) 22:1035-46. doi: 10.1038/cdd.2014.194

144. Aras S. Mitochondrial Autoimmunity and MNRR1 in Breast Carcinogenesis. BMC Cancer (2019) 19:411. doi: 10.1186/s12885-019-5575-7

145. Rosmarin AG, Resendes KK, Yang Z, McMillan JN, Fleming SL. GA-Binding Protein Transcription Factor: A Review of GABP as an Integrator of Intracellular Signaling and Protein-Protein Interactions. Blood Cells $\mathrm{Mol}$ Dis (2004) 32:143-54. doi: 10.1016/j.bcmd.2003.09.005

146. Funayama M, Ohe K, Amo T, Furuya N, Yamaguchi J, Saiki S, et al. CHCHD2 Mutations in Autosomal Dominant Late-Onset Parkinson's Disease: A Genome-Wide Linkage and Sequencing Study. Lancet Neurol (2015) 14:274-82. doi: 10.1016/S1474-4422(14)70266-2

147. White RM, Cech J, Ratanasirintrawoot S, Lin CY, Rahl PB, Burke CJ, et al. DHODH Modulates Transcriptional Elongation in the Neural Crest and Melanoma. Nature (2011) 471:518-22. doi: 10.1038/nature09882

148. Garcia-Bermudez J, Baudrier L, La K, Zhu XG, Fidelin J, Sviderskiy VO, et al. Aspartate is a Limiting Metabolite for Cancer Cell Proliferation Under Hypoxia and in Tumors. Nat Cell Biol (2018) 20:775-81. doi: 10.1038/ s41556-018-0118-z

149. Qian Y, Liang X, Kong P, Cheng Y, Cui H, Yan T, et al. Elevated DHODH Expression Promotes Cell Proliferation via Stabilizing $\beta$-Catenin in Esophageal Squamous Cell Carcinoma. Cell Death Dis (2020) 11:1-13. doi: 10.1038/s41419-020-03044-1

150. Qin J-J, Nag S, Wang W, Zhou J, Zhang W-D, Wang H, et al. NFAT as Cancer Target: Mission Possible? Biochim Biophys Acta (2014) 1846:297311. doi: 10.1016/j.bbcan.2014.07.009

151. Mancini M, Toker A. NFAT Proteins: Emerging Roles in Cancer Progression. Nat Rev Cancer (2009) 9:810-20. doi: 10.1038/nrc2735

152. Zhang X, Zhang Z, Cheng J, Li M, Wang W, Xu W, et al. Transcription Factor NFAT1 Activates the Mdm2 Oncogene Independent of P53. J Biol Chem (2012) 287:30468-76. doi: 10.1074/jbc.M112.373738 
153. Chang ALS, Tran DC, Cannon JGD, Li S, Jeng M, Patel R, et al. Pembrolizumab for Advanced Basal Cell Carcinoma: An InvestigatorInitiated, Proof-of-Concept Study. J Am Acad Dermatol (2019) 80:564-6. doi: 10.1016/j.jaad.2018.08.017

Conflict of Interest: The authors declare that the research was conducted in the absence of any commercial or financial relationships that could be construed as a potential conflict of interest.

Publisher's Note: All claims expressed in this article are solely those of the authors and do not necessarily represent those of their affiliated organizations, or those of the publisher, the editors and the reviewers. Any product that may be evaluated in this article, or claim that may be made by its manufacturer, is not guaranteed or endorsed by the publisher.

Copyright (ㄷ) 2021 Nawrocka, Galka-Marciniak, Urbanek-Trzeciak, MThirusenthilarasan, Szostak, Philips, Susok, Sand and Kozlowski. This is an openaccess article distributed under the terms of the Creative Commons Attribution License (CC BY). The use, distribution or reproduction in other forums is permitted, provided the original author(s) and the copyright owner(s) are credited and that the original publication in this journal is cited, in accordance with accepted academic practice. No use, distribution or reproduction is permitted which does not comply with these terms. 